M. A. Rana • Y. Ali • M. Shoaib

\title{
Three-dimensional Couette flow of a Jeffrey fluid along periodic injection/suction
}

Received: 26 September 2017 / Accepted: 12 March 2018 / Published online: 27 March 2018

(C) The Author(s) 2018

\begin{abstract}
Three-dimensional Couette flow of an incompressible Jeffrey fluid is formulated and discussed analytically and graphically. The suction is applied over uniformly moving upper plate and its equivalent deduction by injection at the lower stationary plate. Because of this type of suction/injection, this flow turns into three-dimensional. An analytical method is applied to get main flow velocity, secondary flows velocities and pressure components. Also skin friction components along the main and secondary flow directions have been calculated. The effects of different physical parameters, for example, the Deborah number, suction/injection parameter, the ratio of relaxation time to the retardation time and Reynolds number have been discussed graphically. It is witnessed that the Deborah number plays vital role to control the main flow velocity.
\end{abstract}

Mathematics Subject Classification $76 \mathrm{~A} 05$

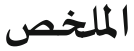

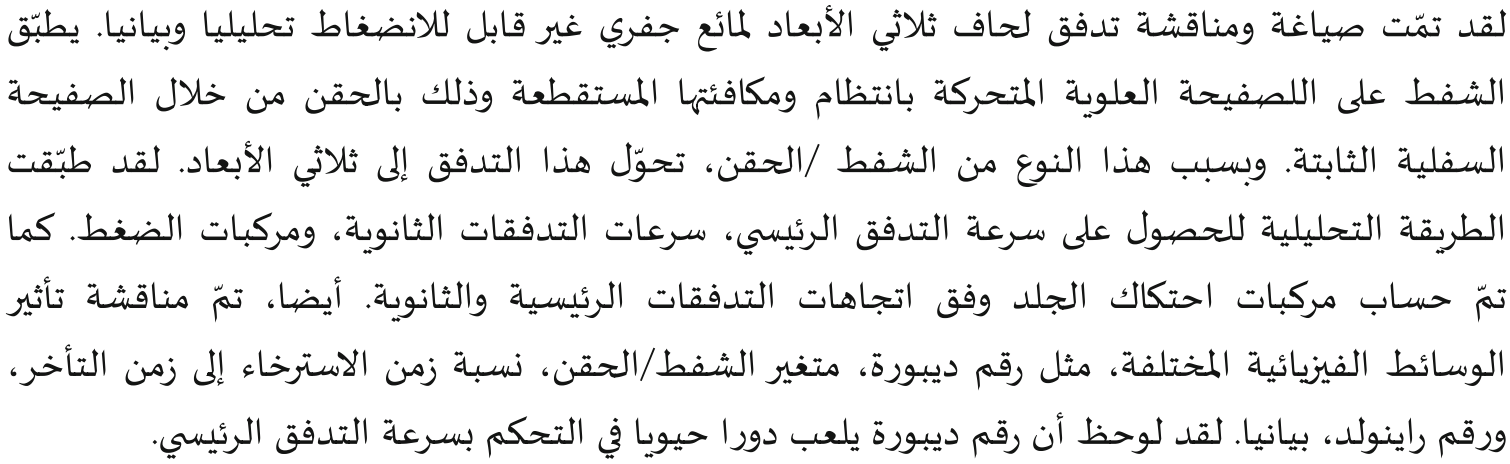

\section{Introduction}

Laminar flow control problems (LFC) have attained considerable value in these days, due to their applications in the reduction of drag and therefore to develop the automobile efficiency significantly. Many techniques

M. A. Rana · Y. Ali (凶)

Department of Mathematics and Statistics, Riphah International University, Islamabad, Pakistan

E-mail: ryasir5@gmail.com

M. Shoaib

COMSATS Institute of Information Technology, Attock, Pakistan 
have been utilized to stable the boundary layer artificially. To reduce the drag coefficient, boundary layer suction technique is an effective method, which causes huge energy losses. Laminarization of boundary layer upon a profile decreases the drag, and hence the automobile efficiency desired by a significant capacity. As stated by boundary layer suction technique, decelerated fluid particles along the boundary layer are separated through the slits and holes in the plane inner side of the body and, thus, the variation from turbulent to laminar flow affecting rise of drag coefficient may be prevented or deferred [1]. Various researchers have taken the different characteristics of fluid flow problems along suction but many of these investigations can handle only two-dimensional flow. Gersten and Gross [2] studied the viscous fluid and observed the effect of heat transfer and sinusoidal transverse suction velocity on flow over a porous plate. Singh [3] considered the problem of transverse periodic suction/injection velocity along transpiration cooling. Chaudhary et al. [4] examined 3D Couette flow along transpiration cooling and stated the effects of injection/suction velocity on the flow field, skin friction and heat transfer. Guria and Jana [5] investigated heat transfer effect on unsteady 3D fluctuating Couette flow between porous plates and found that the main flow velocity decreases with increase in physical parameter, but the secondary flow velocity increases with increase in physical parameter. Sharma et al. [6] studied radiation effect on temperature distribution in three-dimensional Couette flow with suction or injection. It was noted that Prandtl number has a much greater effect than suction/injection parameter on the temperature field. Chauhan [7] examined heat transient effects in a three-dimensional Couette flow through a partly filled channel by a porous material. Various researchers [8-16] also observed three-dimensional flow Newtonian fluid past through porous plates under the influence of different physical parameters.

All the above-mentioned studies have been made in Newtonian fluid. Although the Navier-Stokes equations can handle the flows of Newtonian fluids, these are inadequate to describe the features of non-Newtonian fluids. Shoaib and his co-workers [17] studied three-dimensional flow of Maxwell fluid along an infinite plane wall with the application of periodic suction.

However, in the literature, the Couette flow of a Jeffrey fluid with the application of normal periodic suction/injection velocity has not been studied till now. So, in this work, three-dimensional Couette flow of a Jeffrey fluid with periodic injection/suction is examined. Uniform suction or injection velocity at the plane tends to two-dimensional flow [2], but, due to changing of suction velocity in normal direction on plane the problem converts to three-dimensional flow. The solution is presented using perturbation method. The outcomes achieved are examined for various dimensionless parameters such as Reynolds number Re, Deborah number $\beta$, the ratio of relaxation time to the retardation time $\lambda_{1}$ and injection/suction parameter $\alpha$. The arrangement of the paper is as follows: Section 2 describes the problem, Sect. 3 discusses the problem formulation, Sect. 4 estimates the solutions, Sect. 5 is devoted to the discussion of the results, and Sect. 6 summarizes the conclusions.

\section{Problem description}

Consider Couette flow of an incompressible steady laminar fully developed Jeffrey fluid. The separation between the plates is " $h$ " as shown in Fig. 1. Take the $y^{*}$-axis perpendicular to the plates and the $x^{*} z^{*}-$ plane along the plates. The injection/suction velocity distribution [2] is of the type

$$
v^{*}\left(z^{*}\right)=V_{0}\left(1+\varepsilon \cos \pi \frac{z^{*}}{h}\right)
$$

where $\varepsilon$ is its amplitude and $V_{0}$ is injection/suction velocity. The plate lying down side is fixed, whereas $U$ is constant velocity of higher plate which is moving along the positive $x^{*}$-axis. Consider the transverse periodic suction of the fluid at the higher plate whereas its equivalent elimination by sinusoidal injection at the lower plate. The $u^{*}, v^{*}$ and $w^{*}$ are components of velocity along the $x^{*}-, y^{*}-$ and $z^{*}$-directions, respectively. Since all the quantities are independent of $x^{*}$, therefore, the flow is considered to be fully established and laminar. The flow remains three-dimensional because of variable injection/suction velocity (1).

\section{Problem formulation}

Jeffrey fluid model can be expressed by constitutive equation

$$
\widetilde{T}=-p \widetilde{I}+\widetilde{S},
$$

where

$$
\left.\begin{array}{c}
\mathbf{S}=\frac{\mu}{1+\lambda_{1}}\left(\dot{\mathbf{r}}+\lambda_{2} \ddot{\mathbf{r}}\right), \\
\dot{\mathbf{r}}=\nabla \mathbf{V}+(\nabla \mathbf{V})^{\mathrm{T}}, \ddot{\mathbf{r}}=\frac{\mathrm{d} \dot{\mathbf{r}}}{\mathrm{d} t},
\end{array}\right\}
$$




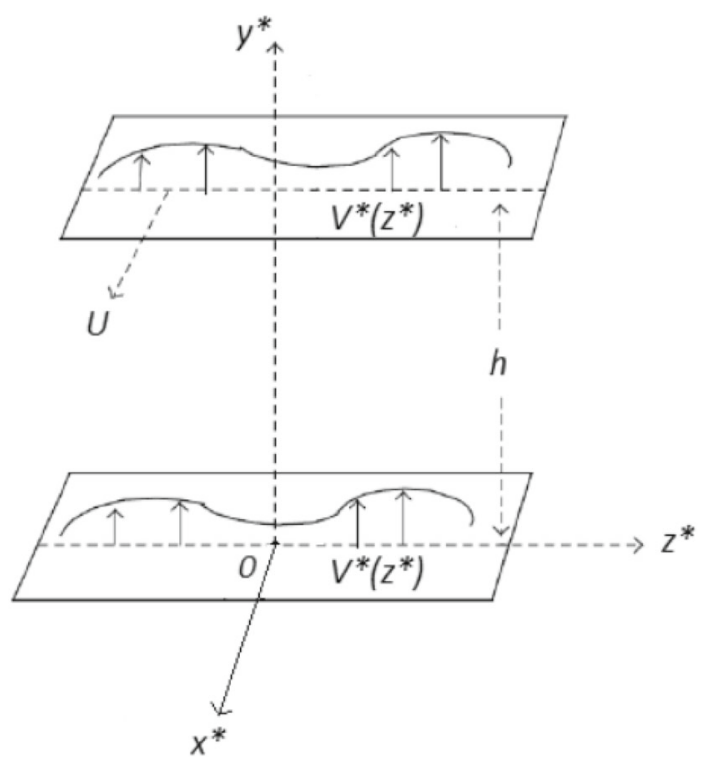

Fig. 1 Schematic of the problem

in which $\mu, p, \lambda_{1}, \lambda_{2}$ and $\widetilde{I}$ denote the dynamic viscosity, the pressure, the ratio of relaxation time to the retardation time, the retardation time and the identity tensor, respectively, where " $\mathrm{T}$ " denotes the transpose. The conservation laws of mass and momentum can be stated as

$$
\begin{aligned}
& \operatorname{div} \mathbf{V}=0, \\
& \rho \frac{\partial \mathbf{V}}{\partial t}=\operatorname{div} \widetilde{T},
\end{aligned}
$$

where $\mathbf{V}$ is velocity profile and $\rho$ represents the fluid density. Thus, following system of partial differential equations governed the given problem:

$$
\begin{aligned}
& \frac{\partial v^{*}}{\partial y^{*}}+\frac{\partial w^{*}}{\partial z^{*}}=0 \\
& \rho\left(v^{*} \frac{\partial u^{*}}{\partial y^{*}}+w^{*} \frac{\partial u^{*}}{\partial z^{*}}\right)=\frac{\mu}{1+\lambda_{1}}\left(\frac{\partial^{2} u^{*}}{\partial y^{* 2}}+\frac{\partial^{2} u^{*}}{\partial z^{* 2}}\right) \\
& +\frac{\mu \lambda_{2}}{1+\lambda_{1}}\left(\begin{array}{c}
\left(v^{*} \frac{\partial}{\partial y^{*}}+w^{*} \frac{\partial}{\partial z^{*}}\right)\left(\left(\frac{\partial^{2} u^{*}}{\partial y^{* 2}}+\frac{\partial^{2} u^{*}}{\partial z^{* 2}}\right)\right) \\
+\left(\frac{\partial v^{*}}{\partial y^{*}} \frac{\partial}{\partial y^{*}}+\frac{\partial w^{*}}{\partial y^{*}} \frac{\partial}{\partial z^{*}}\right) \frac{\partial u^{*}}{\partial y^{*}} \\
+\left(\frac{\partial v^{*}}{\partial z^{*}} \frac{\partial}{\partial y^{*}}+\frac{\partial w^{*}}{\partial z^{*}} \frac{\partial}{\partial z^{*}}\right) \frac{\partial u^{*}}{\partial z^{*}}
\end{array}\right), \\
& \rho\left(v^{*} \frac{\partial v^{*}}{\partial y^{*}}+w^{*} \frac{\partial v^{*}}{\partial z^{*}}\right)=-\frac{\partial p^{*}}{\partial y^{*}}+\frac{\mu}{1+\lambda_{1}}\left(\frac{\partial^{2} v^{*}}{\partial y^{* 2}}+\frac{\partial^{2} v^{*}}{\partial z^{* 2}}\right) \\
& +\frac{\mu \lambda_{2}}{1+\lambda_{1}}\left(\begin{array}{c}
\left(v^{*} \frac{\partial}{\partial y^{*}}+w^{*} \frac{\partial}{\partial z^{*}}\right)\left(\left(\frac{\partial^{2} v^{*}}{\partial y^{* 2}}+\frac{\partial^{2} v^{*}}{\partial z^{* 2}}\right)\right) \\
+2 \frac{\partial v^{*}}{\partial y^{*}} \frac{\partial^{2} u^{*}}{\partial y^{* 2}}+2 \frac{\partial w^{*}}{\partial y^{*}} \frac{\partial^{2} u^{*}}{\partial y^{*} \partial z^{*}}+ \\
\left(\frac{\partial v^{*}}{\partial z^{*}} \frac{\partial}{\partial y^{*}}+\frac{\partial w^{*}}{\partial z^{*}} \frac{\partial}{\partial z^{*}}\right)\left(\frac{\partial v^{*}}{\partial y^{*}}+\frac{\partial w^{*}}{\partial z^{*}}\right)
\end{array}\right), \\
& \rho\left(v^{*} \frac{\partial w^{*}}{\partial y^{*}}+w^{*} \frac{\partial w^{*}}{\partial z^{*}}\right)=-\frac{\partial p^{*}}{\partial z^{*}}+\frac{\mu}{1+\lambda_{1}}\left(\frac{\partial^{2} w^{*}}{\partial y^{* 2}}+\frac{\partial^{2} w^{*}}{\partial z^{* 2}}\right)
\end{aligned}
$$

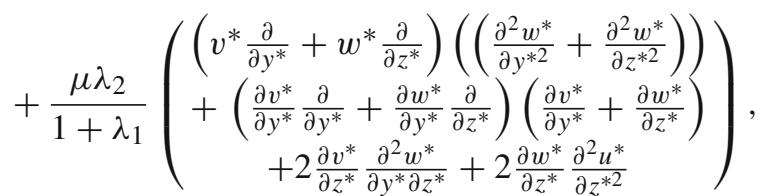


subject to boundary conditions

$$
\left.\begin{array}{l}
\text { at } y^{*}=0 ; u^{*}=0, v^{*}\left(z^{*}\right)=V_{0}\left(1+\varepsilon \cos \pi \frac{z^{*}}{h}\right), w^{*}=0, \\
\text { at } y^{*}=h ; u^{*}=U, v^{*}\left(z^{*}\right)=V_{0}\left(1+\varepsilon \cos \pi \frac{z^{*}}{h}\right), w^{*}=0 .
\end{array}\right\}
$$

Introducing the following dimensionless parameters:

$$
\begin{aligned}
& y=\frac{y^{*}}{h}, \quad z=\frac{z^{*}}{h}, \quad u=\frac{u^{*}}{U}, \quad v=\frac{v^{*}}{U}, w=\frac{w^{*}}{U}, \\
& \alpha=\frac{V_{0}}{U}, \quad \operatorname{Re}=\frac{h U}{v}, \quad R=\left(1+\lambda_{1}\right) \operatorname{Re}, \quad \beta=\frac{\lambda_{2} U}{h}, \quad p=\frac{p^{*}}{\rho U^{2}},
\end{aligned}
$$

then Eqs. (7)-(10) will become

$$
\begin{aligned}
\frac{\partial v}{\partial y}+\frac{\partial w}{\partial z}= & 0 \\
v \frac{\partial u}{\partial y}+w \frac{\partial u}{\partial z}= & \frac{1}{R}\left(\frac{\partial^{2} u}{\partial y^{2}}+\frac{\partial^{2} u}{\partial z^{2}}\right) \\
& +\frac{\beta}{R}\left(\begin{array}{c}
\left(v \frac{\partial}{\partial y}+w \frac{\partial}{\partial z}\right)\left(\frac{\partial^{2} u}{\partial y^{2}}+\frac{\partial^{2} u}{\partial z^{2}}\right) \\
+\left(\frac{\partial v}{\partial y} \frac{\partial}{\partial y}+\frac{\partial w}{\partial y} \frac{\partial}{\partial z}\right) \frac{\partial u}{\partial y}+\left(\frac{\partial v}{\partial z} \frac{\partial}{\partial y}+\frac{\partial w}{\partial z} \frac{\partial}{\partial z}\right) \frac{\partial u}{\partial z}
\end{array}\right), \\
v \frac{\partial v}{\partial y}+w \frac{\partial v}{\partial z}= & -\frac{\partial p}{\partial y}+\frac{1}{R}\left(\frac{\partial^{2} v}{\partial y^{2}}+\frac{\partial^{2} v}{\partial z^{2}}\right) \\
& +\frac{\beta}{R}\left(\begin{array}{c}
\left(v \frac{\partial}{\partial y}+w \frac{\partial}{\partial z}\right)\left(\frac{\partial^{2} v}{\partial y^{2}}+\frac{\partial^{2} v}{\partial z^{2}}\right)+2 \frac{\partial v}{\partial y} \frac{\partial^{2} u}{\partial y^{2}} \\
+2 \frac{\partial w}{\partial y} \frac{\partial^{2} u}{\partial y \partial z}+\left(\frac{\partial v}{\partial z} \frac{\partial}{\partial y}+\frac{\partial w}{\partial z} \frac{\partial}{\partial z}\right)\left(\frac{\partial v}{\partial y}+\frac{\partial w}{\partial z}\right)
\end{array}\right), \\
v \frac{\partial w}{\partial y}+w \frac{\partial w}{\partial z}= & -\frac{\partial p}{\partial z}+\frac{1}{R}\left(\frac{\partial^{2} w}{\partial y^{2}}+\frac{\partial^{2} w}{\partial z^{2}}\right) \\
& +\frac{\beta}{R}\left(\begin{array}{c}
\left(v \frac{\partial}{\partial y}+w \frac{\partial}{\partial z}\right)\left(\frac{\partial^{2} w}{\partial y^{2}}+\frac{\partial^{2} w}{\partial z^{2}}\right)+2 \frac{\partial v}{\partial z} \frac{\partial^{2} u}{\partial y \partial z} \\
+2 \frac{\partial w}{\partial z} \frac{\partial^{2} u}{\partial z^{2}}+\left(\frac{\partial v}{\partial y} \frac{\partial}{\partial y}+\frac{\partial w}{\partial y} \frac{\partial}{\partial z}\right)\left(\frac{\partial v}{\partial y}+\frac{\partial w}{\partial z}\right)
\end{array}\right),
\end{aligned}
$$

subject to dimensionless boundary conditions

$$
\left.\begin{array}{l}
\text { at } y=0 ; u=0, v(z)=\alpha(1+\varepsilon \cos \pi z), w=0, \\
\text { at } y=1 ; u=1, v(z)=\alpha(1+\varepsilon \cos \pi z), w=0 .
\end{array}\right\}
$$

Here $u, v$ and $w$ represent the velocity components in the $x-, y-$ and $z-$ directions, respectively.

\section{Solution}

In this section, the solutions for the velocity field and skin friction components are calculated.

\subsection{Cross-flow solution}

Since $0 \leq \varepsilon<<1$, hence consider the following type of solution

$$
L(y, z)=L_{0}(y)+\varepsilon L_{1}(y, z)+\varepsilon^{2} L_{2}(y, z)+\cdots,
$$

where $L$ stands for any of $u, v, w$ and $p$. As set of the cross-flow solutions $v_{1}(y, z), w_{1}(y, z)$ and $p_{1}(y, z)$ are free from $u$ the main flow velocity component. The differential equations of motion leading the fluid flow are

$$
\frac{\partial v_{1}}{\partial y}+\frac{\partial w_{1}}{\partial z}=0
$$




$$
\begin{gathered}
\alpha R \frac{\partial v_{1}}{\partial y}=-R \frac{\partial p_{1}}{\partial y}+\frac{\partial^{2} v_{1}}{\partial y^{2}}+\frac{\partial^{2} v_{1}}{\partial z^{2}}+\beta \alpha\left(\frac{\partial^{3} v_{1}}{\partial y^{3}}+\frac{\partial^{3} v_{1}}{\partial y \partial z^{2}}\right), \\
\alpha \frac{\partial w_{1}}{\partial y}=-R \frac{\partial p_{1}}{\partial z}+\frac{\partial^{2} w_{1}}{\partial y^{2}}+\frac{\partial^{2} w_{1}}{\partial z^{2}}+\beta \alpha\left(\frac{\partial^{3} w_{1}}{\partial y^{3}}+\frac{\partial^{3} w_{1}}{\partial y \partial z^{2}}\right),
\end{gathered}
$$

and the boundary conditions are

$$
v_{1}(0, z)=\alpha \cos \pi z, w_{1}(0, z)=0, v_{1}(1, z)=\alpha \cos \pi z, w_{1}(1, z)=0 .
$$

The suction/injection velocity comprises basic constant distribution $v_{o}$ along a weak superimposed periodic distribution $\varepsilon v_{o} \cos \pi z$, and hence the components of the velocity $v_{1}(y, z), w_{1}(y, z)$ and pressure $p_{1}(y, z)$ are also detached into small and main periodic components. Therefore, consider the following

$$
\begin{aligned}
& v_{1}(y, z)=v_{11}(y) \cos \pi z, \\
& w_{1}(y, z)=-\frac{1}{\pi} v_{11}^{\prime} \sin \pi z, \\
& p_{1}(y, z)=p_{11}(y) \cos \pi z .
\end{aligned}
$$

Here " " " represents the differentiation w.r.t " $y$ ". It is stating that the components of velocity (23)-(24) identically fulfil the continuity Eq. ( 19). Substituting Eqs. (23)-(25) into Eqs. (20) and (21) to get

$$
\begin{aligned}
& v_{11}^{\prime \prime \prime}-\pi^{2} v_{11}^{\prime}-\alpha R v_{11}^{\prime \prime}+\beta \alpha\left(v_{11}^{\prime v}-\pi^{2} v_{11}^{\prime \prime}\right)=R \pi^{2} p_{11}, \\
& v_{11}^{\prime \prime}-\alpha R v_{11}^{\prime}-\pi^{2} v_{11}+\beta \alpha\left(v_{11}^{\prime \prime \prime}-\pi^{2} v_{11}^{\prime}\right)=R p_{11}^{\prime} .
\end{aligned}
$$

Eliminating the pressure $p_{11}$ from Eqs. (26) and (27) to get

$$
v_{11}^{\prime v}-\alpha R\left(v_{11}^{\prime \prime \prime}-\pi^{2} v_{11}^{\prime}\right)-2 \pi^{2} v_{11}^{\prime \prime}+\pi^{4} v_{11}=-\beta \alpha\left(v_{11}^{v}-2 \pi^{2} v_{11}^{\prime \prime \prime}+\pi^{4} v_{11}^{\prime}\right) .
$$

Assuming $\beta<<1$, and taking

$$
v_{11}(y)=v_{110}(y)+\beta v_{111}(y)+O\left(\beta^{2}\right),
$$

then Eq. (28) yields the zeroth-order equation given by

$$
v_{110}^{\prime v}-\alpha R\left(v_{110}^{\prime \prime \prime}-\pi^{2} v_{110}^{\prime}\right)-2 \pi^{2} v_{110}^{\prime \prime}+\pi^{4} v_{110}=0 .
$$

Subjected to the boundary conditions

$$
v_{110}(0)=\alpha=v_{110}(1), v_{110}^{\prime}(0)=0=v_{110}^{\prime}(1) .
$$

The solution of Eqs. (30) and (31) yields

$$
v_{110}(y)=S_{3} \mathrm{e}^{-\pi y}+S_{4} \mathrm{e}^{\pi y}+S_{5} \mathrm{e}^{S_{1} y}+S_{6} \mathrm{e}^{S_{2} y} .
$$

Similarly, the first-order equation is

$$
v_{111}^{\prime v}+\pi^{4} v_{111}-2 \pi^{2} v_{111}^{\prime \prime}-\alpha R\left(v_{111}^{\prime \prime \prime}-\pi^{2} v_{111}^{\prime}\right)=-\alpha\left(v_{110}^{v}-2 \pi^{2} v_{110}^{\prime \prime \prime}+\pi^{4} v_{110}^{\prime}\right),
$$

and the boundary conditions are

$$
v_{111}(0)=0, v_{111}(1)=0, v_{111}^{\prime}(0)=0, v_{111}^{\prime}(1)=0 .
$$

The solution of the BVP (33) and (34) is

$$
v_{111}(y)=S_{7} \mathrm{e}^{-\pi y}+S_{8} \mathrm{e}^{\pi y}+S_{9} \mathrm{e}^{S_{1} y}+S_{10} \mathrm{e}^{S_{2} y}+y\left(S_{11} \mathrm{e}^{S_{1} y}+S_{12} \mathrm{e}^{S_{2} y}\right) .
$$

Thus, Eqs. (29), (23) and (24), respectively, become

$$
\begin{aligned}
v_{11}(y)= & S_{3} \mathrm{e}^{-\pi y}+S_{4} \mathrm{e}^{\pi y}+S_{5} \mathrm{e}^{S_{1} y}+S_{6} \mathrm{e}^{S_{2} y} \\
& +\beta\left(S_{7} \mathrm{e}^{-\pi y}+S_{8} \mathrm{e}^{\pi y}+S_{9} \mathrm{e}^{S_{1} y}+S_{10} \mathrm{e}^{S_{2} y}+y\left(S_{11} \mathrm{e}^{S_{1} y}+S_{12} \mathrm{e}^{S_{2} y}\right)\right),
\end{aligned}
$$




$$
\begin{aligned}
v_{1}(y, z) & =\left(\begin{array}{c}
S_{3} \mathrm{e}^{-\pi y}+S_{4} \mathrm{e}^{\pi y}+S_{5} \mathrm{e}^{S_{1} y}+S_{6} \mathrm{e}^{S_{2} y}+ \\
\beta\left(\begin{array}{c}
S_{7} \mathrm{e}^{-\pi y}+S_{8} \mathrm{e}^{\pi y}+S_{9} \mathrm{e}_{1} y \\
S_{10} \mathrm{e}^{S_{2} y}+y\left(S_{11} \mathrm{e}^{S_{1} y}+S_{12} \mathrm{e}^{S_{2} y}\right.
\end{array}\right)
\end{array}\right) \cos \pi z, \\
w_{1}(y, z) & =-\frac{1}{\pi}\left(\begin{array}{c}
-S_{3} \pi \mathrm{e}^{-\pi y}+S_{4} \pi \mathrm{e}^{\pi y}+S_{5} S_{1} \mathrm{e}^{S_{1} y}+S_{6} S_{2} \mathrm{e}^{S_{2} y}+ \\
\beta\left(\begin{array}{c}
-S_{7} \pi \mathrm{e}^{-\pi y}+S_{8} \pi \mathrm{e}^{\pi y}+S_{9} S_{1} \mathrm{e}^{S_{1} y}+S_{10} S_{2} \mathrm{e}^{S_{2} y}+ \\
S_{11} \mathrm{e}^{S_{1} y}+S_{12} \mathrm{e}^{S_{2} y}+y\left(S_{11} S_{1} \mathrm{e}^{S_{1} y}+S_{12} S_{2} \mathrm{e}^{S_{2} y}\right)
\end{array}\right)
\end{array}\right) \sin \pi z,
\end{aligned}
$$

where constants $S_{i}(i=1,2,3, \ldots, 12)$ are defined in "Appendix A".

\subsection{Main flow solution}

When $\varepsilon=0$, the problem becomes two-dimensional flow, and hence

$$
\frac{\mathrm{d}^{2} u_{0}}{\mathrm{~d} y^{2}}-\alpha R \frac{\mathrm{d} u_{0}}{\mathrm{~d} y}=-\beta \alpha \frac{\mathrm{d}^{3} u_{0}}{\mathrm{~d} y^{3}},
$$

and boundary conditions are

$$
u_{0}(0)=0, u_{0}(1)=1 \text {. }
$$

Since $\beta<<1$, so assuming

$$
u_{0}(y)=u_{00}(y)+\beta u_{01}(y)+O\left(\beta^{2}\right),
$$

the solution of the zeroth-order BVP

$$
\begin{aligned}
& \frac{\mathrm{d}^{2} u_{00}}{\mathrm{~d} y^{2}}-\alpha R \frac{\mathrm{d} u_{00}}{\mathrm{~d} y}=0, \\
& u_{00}(0)=0, u_{00}(1)=1 .
\end{aligned}
$$

is

$$
u_{00}(y)=1+\frac{\mathrm{e}^{\alpha R y}-\mathrm{e}^{\alpha R}}{\mathrm{e}^{\alpha R}-1} .
$$

Similarly, solution of the first-order BVP

$$
\left.\begin{array}{c}
\frac{\mathrm{d}^{2} u_{01}}{\mathrm{~d} y^{2}}-\alpha R \frac{\mathrm{d} u_{01}}{\mathrm{~d} y}=-\alpha \frac{\mathrm{d}^{3} u_{00}}{\mathrm{~d} y^{3}}, \\
u_{01}(0)=0, u_{01}(1)=0,
\end{array}\right\}
$$

is

$$
u_{01}(y)=S_{13}+S_{14} \mathrm{e}^{\alpha R y}+y S_{15} \mathrm{e}^{\alpha R y} .
$$

Thus

$$
u_{0}(y)=1+\frac{\mathrm{e}^{\alpha R y}-\mathrm{e}^{\alpha R}}{\mathrm{e}^{\alpha R}-1}+\beta\left(S_{13}+S_{14} \mathrm{e}^{\alpha R y}+y S_{15} \mathrm{e}^{\alpha R y}\right),
$$

where the constants $S_{13}, S_{14}$ and $S_{15}$ are given in "Appendix A". The case when $\varepsilon \neq 0$, the differential equations (14)-(16) governing the fluid flow and boundary conditions (17) are perturbed by taking

$$
\begin{gathered}
u(y, z)=u_{0}(y)+\varepsilon u_{1}(y, z)+O\left(\varepsilon^{2}\right), \\
v(y, z)=v_{0}(y)+\varepsilon v_{1}(y, z)+O\left(\varepsilon^{2}\right), \\
w(y, z)=w_{0}(y)+\varepsilon w_{1}(y, z)+O\left(\varepsilon^{2}\right) .
\end{gathered}
$$

Then the first-order equation

$$
\alpha R \frac{\partial u_{1}}{\partial y}+v_{1} R \frac{\mathrm{d} u_{0}}{\mathrm{~d} y}=\frac{\partial^{2} u_{1}}{\partial y^{2}}+\frac{\partial^{2} u_{1}}{\partial z^{2}}+\beta\left(\begin{array}{c}
\alpha\left(\frac{\partial^{3} u_{1}}{\partial y^{3}}+\frac{\partial^{3} u_{1}}{\partial y \partial z^{2}}\right) \\
+v_{1} \frac{\mathrm{d}^{3} u_{0}}{\mathrm{~d} y^{3}}+\frac{\partial v_{1}}{\partial y} \frac{\mathrm{d}^{2} u_{0}}{\mathrm{~d} y^{2}}
\end{array}\right),
$$

and corresponding boundary conditions are

$$
u_{1}(0, z)=0=u_{1}(1, z) .
$$


The solution of Eq. (52) can be expressed as $u_{1}(y, z)=u_{11}(y) \cos \pi z$. Then

$$
\frac{\mathrm{d}^{2} u_{11}}{\mathrm{~d} y^{2}}-\pi^{2} u_{11}-\alpha R\left(\frac{\mathrm{d} u_{11}}{\mathrm{~d} y}+v_{11} \frac{\mathrm{d} u_{0}}{\mathrm{~d} y}\right)=-\beta\left(\begin{array}{c}
\alpha\left(\frac{\mathrm{d}^{3} u_{11}}{\mathrm{~d} y^{3}}-\pi^{2} \frac{\mathrm{d} u_{11}}{\mathrm{~d} y}\right) \\
+v_{11} \frac{\mathrm{d}^{3} u_{0}}{\mathrm{~d} y^{3}}+\frac{\mathrm{d} v_{11}}{\mathrm{~d} y} \frac{\mathrm{d}^{2} u_{0}}{\mathrm{~d} y^{2}}
\end{array}\right),
$$

and the boundary conditions (53) will become

$$
u_{11}(0)=0=u_{11}(1) .
$$

We have third-order Eq. (54), whereas we have two boundary conditions only. Therefore, we express the solution of Eq. (54) as follows:

$$
u_{11}(y)=u_{110}(y)+\beta u_{111}(y)+O\left(\beta^{2}\right) .
$$

Then the solution of zeroth-order problem

$$
\begin{aligned}
& \frac{\mathrm{d}^{2} u_{110}}{\mathrm{~d} y^{2}}-\alpha R \frac{\mathrm{d} u_{110}}{\mathrm{~d} y}-\pi^{2} u_{110}=\alpha R v_{110} \frac{\mathrm{d} u_{00}}{\mathrm{~d} y}, \\
& u_{110}(0)=0=u_{110}(1)
\end{aligned}
$$

is

$$
u_{110}(y)=S_{16} \mathrm{e}^{S_{1} y}+S_{17} \mathrm{e}^{S_{2} y}+S_{18} \mathrm{e}^{(\alpha R-\pi) y}+S_{19} \mathrm{e}^{(\alpha R+\pi) y}+S_{20} \mathrm{e}^{\left(\alpha R+S_{1}\right) y}+S_{21} \mathrm{e}^{\left(\alpha R+S_{2}\right) y} .
$$

Similarly, the solution of first-order problem and corresponding boundary conditions

$$
\begin{aligned}
& \frac{\mathrm{d}^{2} u_{111}}{\mathrm{~d} y^{2}}-\alpha R \frac{\mathrm{d} u_{111}}{\mathrm{~d} y}-\pi^{2} u_{111} \\
& =\alpha R\left(v_{111} \frac{\mathrm{d} u_{00}}{\mathrm{~d} y}+v_{110} \frac{\mathrm{d} u_{01}}{\mathrm{~d} y}\right)-\alpha\left(\frac{\mathrm{d}^{3} u_{110}}{\mathrm{~d} y^{3}}-\pi^{2} \frac{\mathrm{d} u_{110}}{\mathrm{~d} y}\right)+v_{110} \frac{\mathrm{d}^{3} u_{00}}{\mathrm{~d} y^{3}}+\frac{\mathrm{d} v_{110}}{\mathrm{~d} y} \frac{\mathrm{d}^{2} u_{00}}{\mathrm{~d} y^{2}}, \\
& u_{111}(0)=0=u_{111}(1)
\end{aligned}
$$

is

$$
\begin{aligned}
& S_{16} \mathrm{e}^{S_{1} y}+S_{17} \mathrm{e}^{S_{2} y}+S_{18} \mathrm{e}^{(\alpha R-\pi) y}+S_{19} \mathrm{e}^{(\alpha R+\pi) y}+S_{20} \mathrm{e}^{\left(\alpha R+S_{1}\right) y}+S_{21} \mathrm{e}^{\left(\alpha R+S_{2}\right) y}
\end{aligned}
$$

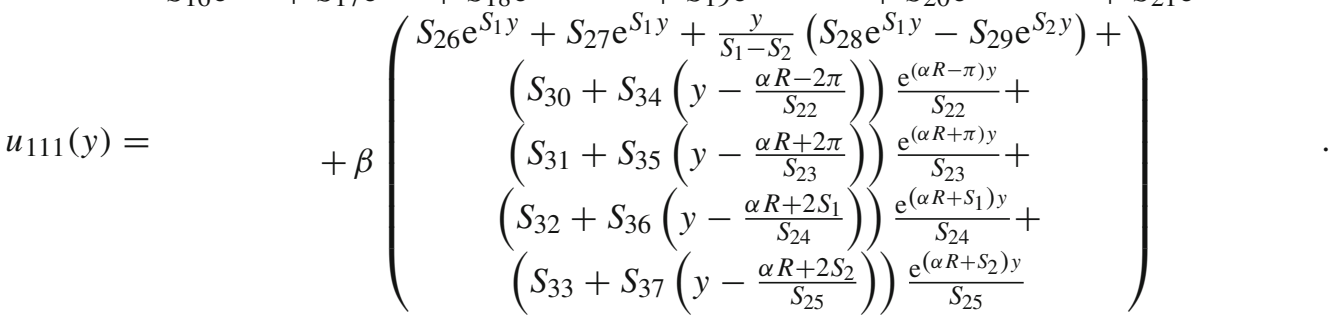

In view of Eqs. (47), (58), (61) and (55), Eq. (48) yields

$$
\begin{aligned}
& u(y, z)=1+\frac{\mathrm{e}^{\alpha R y}-\mathrm{e}^{\alpha R}}{\mathrm{e}^{\alpha R}-1}+\beta\left(S_{13}+S_{14} \mathrm{e}^{\alpha R y}+y S_{15} \mathrm{e}^{\alpha R y}\right)
\end{aligned}
$$

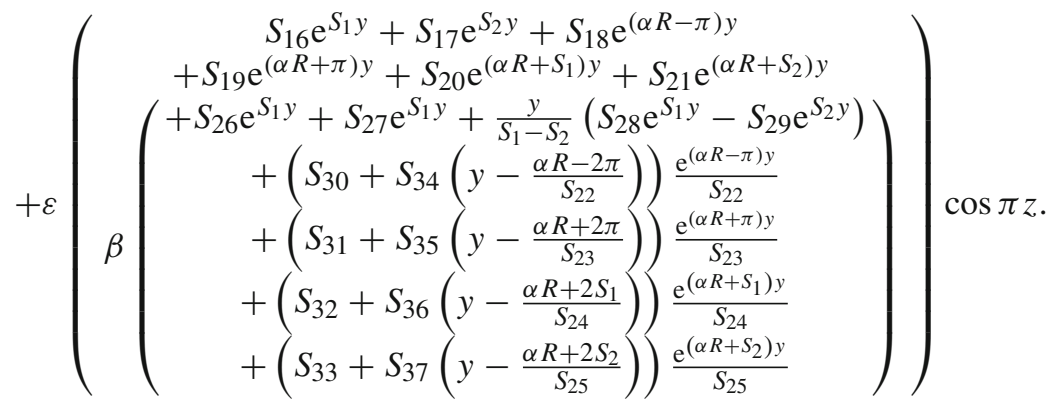




\subsection{Skin friction factors}

The components of skin friction $F_{1}$ and $F_{2}$ in the $x$-direction and $z$-direction, respectively, are given below:

$$
\left.\begin{array}{c}
\tau_{x}=\left(\frac{\mathrm{d} u_{0}}{\mathrm{~d} y}\right)_{y=0}+\varepsilon\left(\frac{\mathrm{d} u_{11}}{\mathrm{~d} y}\right)_{y=0} \cos \pi z, \\
\tau_{z}=\frac{-\varepsilon}{\pi}\left(\frac{\mathrm{d} v_{11}^{\prime}}{\mathrm{d} y}\right)_{y=0} \sin \pi z, \\
F_{1}=\left(\frac{\mathrm{d} u_{11}}{\mathrm{~d} y}\right)_{y=0}, F_{2}=\frac{-1}{\pi}\left(\frac{\mathrm{d} v_{11}^{\prime}}{\mathrm{d} y}\right)_{y=0} .
\end{array}\right\}
$$

So Eq. (38) yields

$$
F_{2}=-\frac{1}{\pi}\left(\begin{array}{c}
S_{3} \pi^{2}+S_{4} \pi^{2}+S_{5} S_{1}^{2}+S_{6} S_{2}^{2} \\
+\beta\left(S_{7} \pi^{2}+S_{8} \pi^{2}+S_{9} S_{1}^{2}+S_{10} S_{2}^{2}+2 S_{11} S_{1}+2 S_{12} S_{2}\right)
\end{array}\right) .
$$

Similarly, Eqs. (55), (58) and (61) yield

$$
\begin{aligned}
F_{1}= & S_{1} S_{16}+S_{2} S_{17}+S_{20}\left(S_{1}+\alpha R\right) \\
& +S_{21}\left(S_{2}+\alpha R\right)+S_{18}(\alpha R-\pi)+S_{19}(\alpha R+\pi) \\
& +\beta\left(\begin{array}{c}
S_{1} S_{26}+S_{2} S_{27}+\frac{S_{28} \mathrm{~S}_{1}}{S_{1}-S_{2}}+\frac{S_{29} S_{2}}{S_{2}-S_{1}}+\frac{S_{30}(\alpha R-\pi)}{S_{22}} \\
+\frac{S_{31}(\alpha R+\pi)}{S_{23}}+\frac{S_{22}\left(\alpha R+S_{1}\right)}{S_{24}}+\frac{S_{33}\left(\alpha R+S_{2}\right)}{S_{25}} \\
+\frac{S_{34}}{S_{22}}\left(1-\frac{(\alpha R-\pi)(\alpha R-2 \pi)}{S_{22}}\right)+\frac{S_{35}}{S_{23}}\left(1-\frac{(\alpha R+\pi)(\alpha R+2 \pi)}{S_{23}}\right) \\
+\frac{S_{36}}{S_{24}}\left(1-\frac{\left(\alpha R+S_{1}\right)\left(\alpha R+2 S_{1}\right)}{S_{24}}\right)+\frac{S_{37}}{S_{25}}\left(1-\frac{\left(\alpha R+S_{2}\right)\left(\alpha R+2 S_{2}\right)}{S_{25}}\right)
\end{array}\right) .
\end{aligned}
$$

\subsection{Pressure}

Substituting Eq. (36) in Eq. (27) and simplifying to obtain

$$
\begin{aligned}
p_{11}(y)= & S_{38} \mathrm{e}^{-\pi y}+S_{39} \mathrm{e}^{\pi y}+S_{40} \mathrm{e}^{S_{1} y}+S_{41} \mathrm{e}^{S_{2} y} \\
& +\beta\left(\begin{array}{c}
S_{42} \mathrm{e}^{-\pi y}+S_{43} \mathrm{e}^{\pi y}+S_{44} \mathrm{e}^{S_{1} y} \\
+S_{45} \mathrm{e}^{S_{2} y}+y\left(S_{46} \mathrm{e}^{S_{1} y}+S_{47} \mathrm{e}^{S_{2} y}\right)
\end{array}\right) .
\end{aligned}
$$

The constants $S_{i}(i=16,17,18, \ldots, 47)$ are defined in "Appendix A".

\section{Discussion and results}

In this study, a steady and incompressible fully established and laminar Couette flow of Jeffrey fluid with sinusoidal suction/injection is modelled and examined. The lower plate is still, while higher plate moves along the positive $x$-axis with constant velocity $U$. The transverse sinusoidal suction of the fluid through the upper plate whereas its corresponding removal by sinusoidal injection at the lower plate is taken. Because of the application of variable transverse periodic suction/injection velocity at the plates, this flow becomes three-dimensional. The equations of motion are solved through regular perturbation method. The effects of various non-dimensional physical parameters on components of velocity, skin friction and pressure are shown in Figs. 2, 3, 4, 5, 6, 7, 8, 9, 10, 11, 12, 13, 14, 15, 16, 17, 18, 19, 20, 21, 22 and 23 graphically.

The components of main flow velocity profiles are illustrated in Figs. 2, 3, 4 and 5. The effects of injection/suction parameter $\alpha$, the Deborah number $\beta$, the ratio of relaxation time to the retardation time $\lambda_{1}$ and Reynolds number Re are shown in Figs. 2, 3, 4 and 5, respectively. It is noted that the main flow velocity decreases exponentially along growing injection/suction parameter, the ratio of relaxation time to the retardation time $\lambda_{1}$ or Reynolds number Re. In fact, fluid experience greater viscosity with the porous boundaries and hence offers resistance to flow resulting reduction in the velocity. For large values of injection/suction, the ratio of relaxation time to the retardation time or Reynolds number, the decay is more. However, the main flow velocity increases exponentially with increase in the Deborah number as shown in Fig. 3. The maximum and minimum velocities arise on the plates, which are the velocities of the plates. 


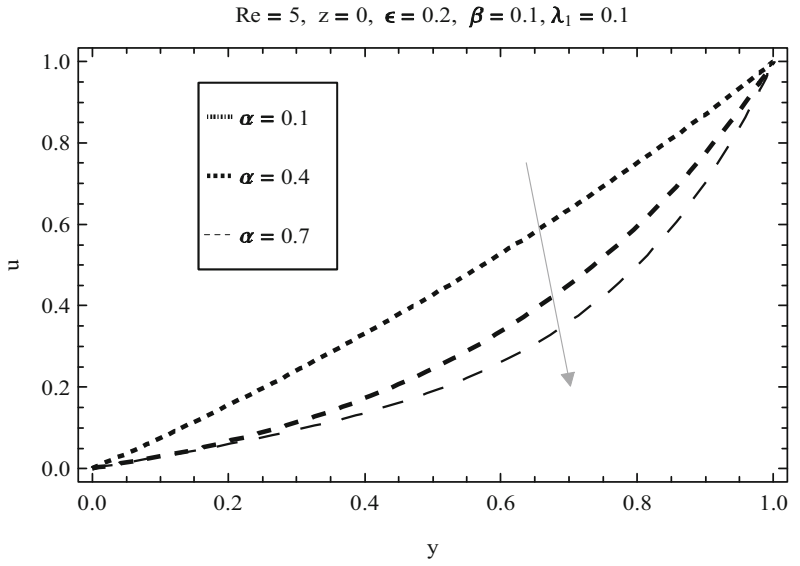

Fig. 2 Velocity component $u$ along $y$ for different values of $\alpha$

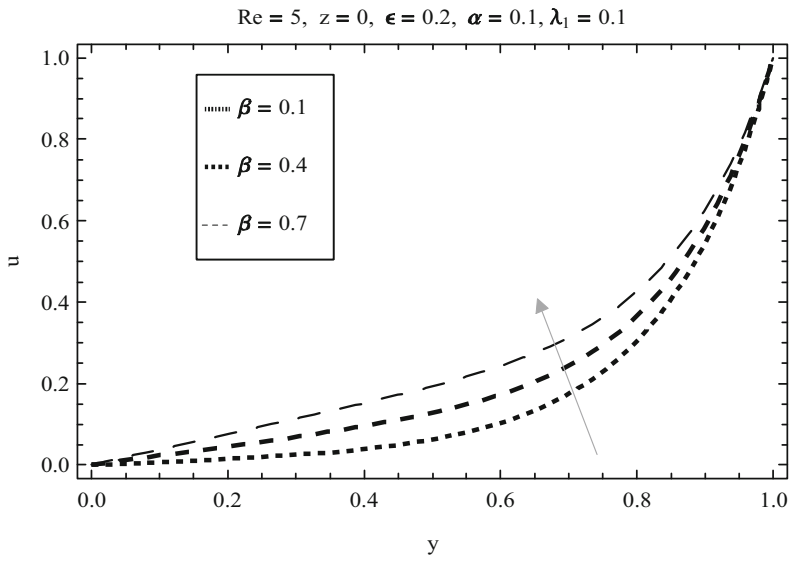

Fig. 3 Velocity component $u$ along $y$ for different values of $\beta$

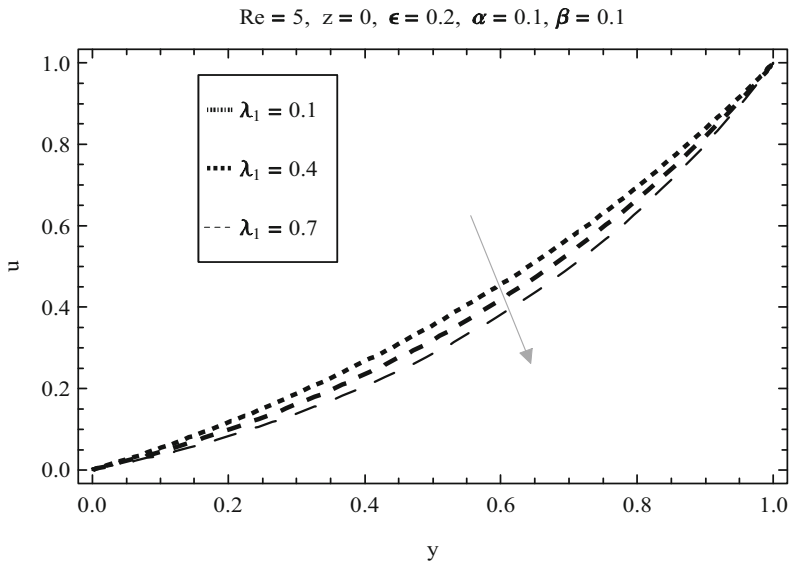

Fig. 4 Velocity component $u$ along $y$ for different values of $\lambda_{1}$

The influence of injection/suction parameter $\alpha$, the Deborah number $\beta$, the ratio of relaxation time to the retardation time $\lambda_{1}$ and Reynolds number Re on secondary flow velocity component $v$ are shown in Figs. 6, 7, 8 and 9, respectively. It is observed that the velocity $v$ increases with increasing the injection/suction parameter, the Deborah number, the ratio of relaxation time to the retardation time and Reynolds number. It means that all the physical parameters provide a mechanism to enhance the velocity $v$. Moreover, the velocity profile behaves 


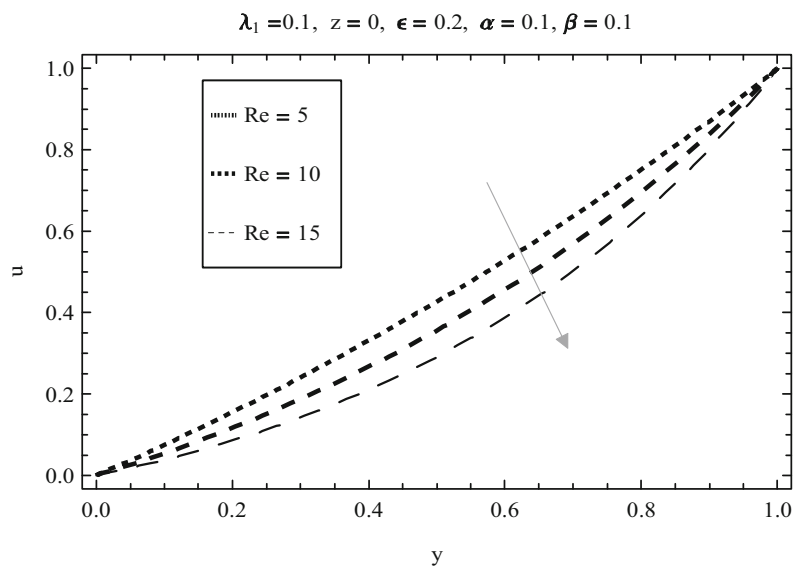

Fig. 5 Velocity component $u$ along $y$ for different values of Re

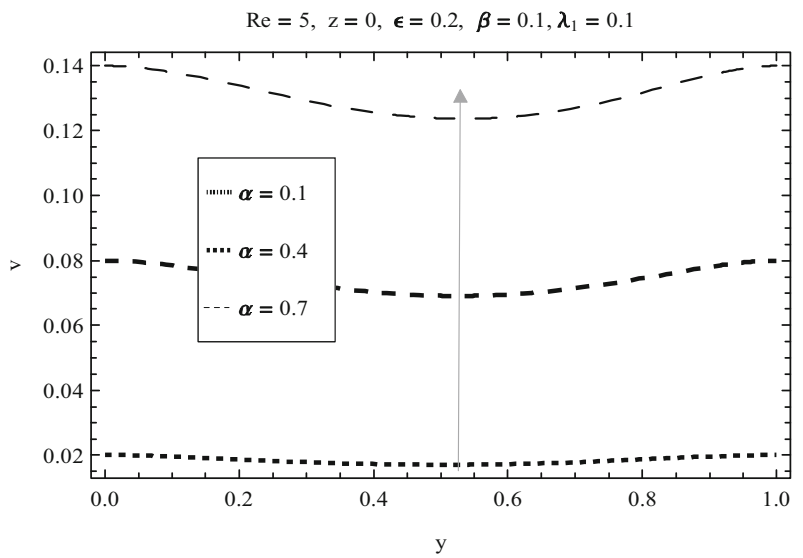

Fig. 6 Velocity component $v$ along $y$ for different values of $\alpha$

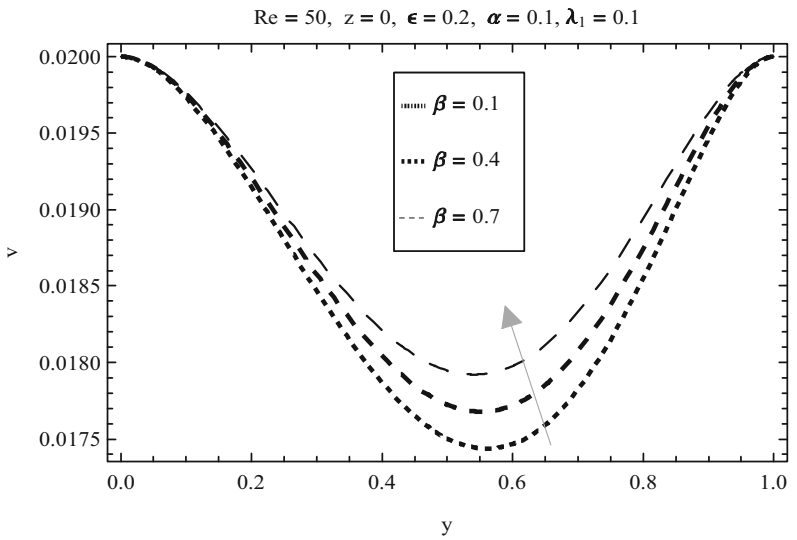

Fig. 7 Velocity component $v$ along $y$ for different values of $\beta$

as a linear function for $\lambda_{1}$ and Re near the upper moving plate as shown in Figs. 8 and 9. Symmetric velocity profiles about the mid of the plates are obtained.

The transverse velocity component $w$ is studied for different values of injection/suction parameter $\alpha$, the Deborah number $\beta$, the ratio of relaxation time to the retardation time $\lambda_{1}$ and Reynolds number Re in Figs. 10, 11,12 and 13. It is noted that forward flow is developed from $y=0$ to about $y=0.5$, and then, onwards, there is backward flow. In fact, the dragging effect of the faster layer exerted on the fluid particles in the 


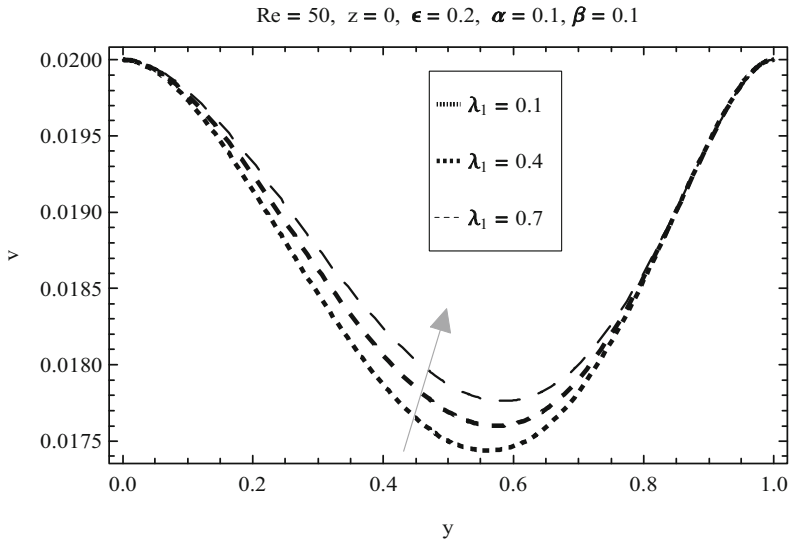

Fig. 8 Velocity component $v$ along $y$ for different values of $\lambda_{1}$

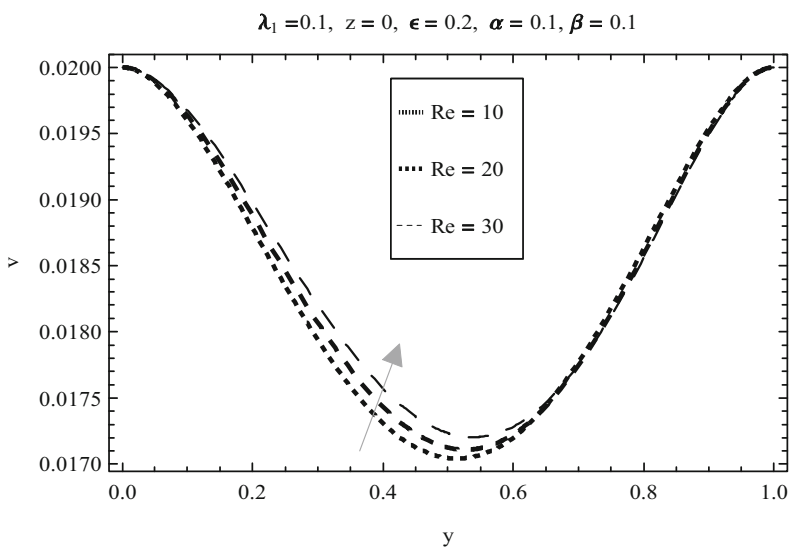

Fig. 9 Velocity component $v$ along $y$ for different values of Re

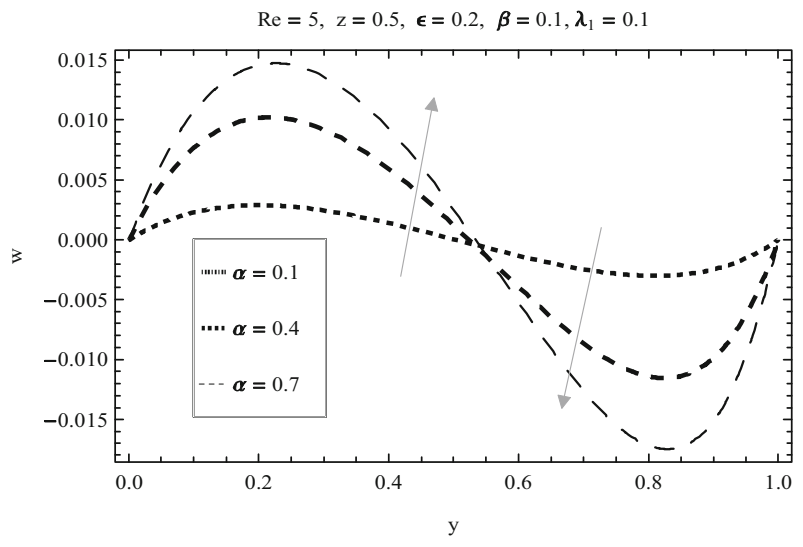

Fig. 10 Velocity component $w$ along $y$ for different values of $\alpha$

neighbourhood of the lower plate (stationary plate) is sufficient to overcome the adverse pressure gradient, and hence there is forward flow. On the contrary, due to the periodic suction at the upper plate (moving plate), the dragging effect of the faster layer exerted on the fluid particles will be reduced, and hence this dragging effect is insufficient to overcome the adverse pressure gradient and there is backflow. From Fig. 10, it is shown that the velocity component $w$ increases with an increase of $\alpha$ in forward flow, but decreases with an increase of $\alpha$ in backward flow. It is obvious from Figs. 11, 12 and 13 that the velocity component $w$ decreases with an increase of $\beta, \lambda_{1}$ or Re in forward flow; however, a reverse effect is seen in the backward flow. 


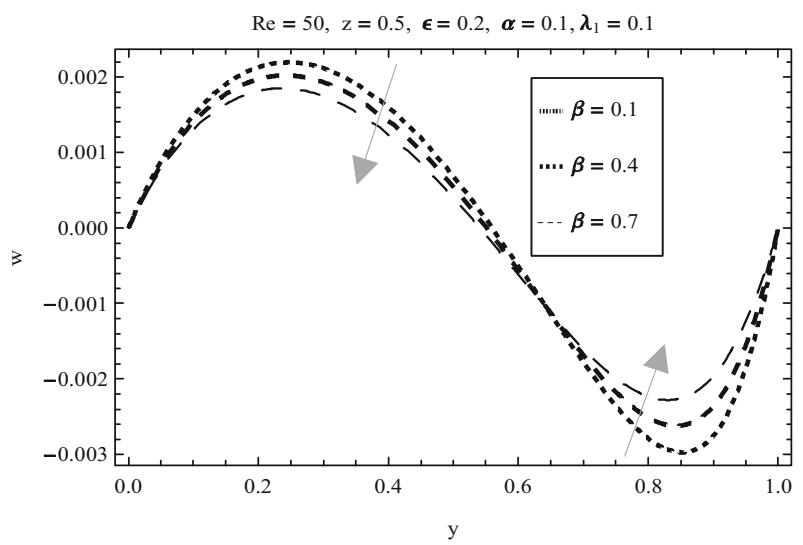

Fig. 11 Velocity component $w$ along $y$ for different values of $\beta$

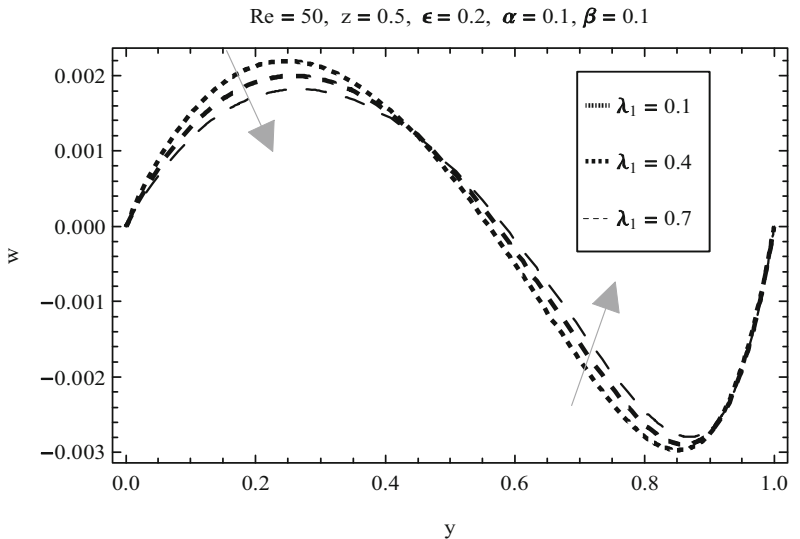

Fig. 12 Velocity component $w$ along $y$ for different values of $\lambda_{1}$

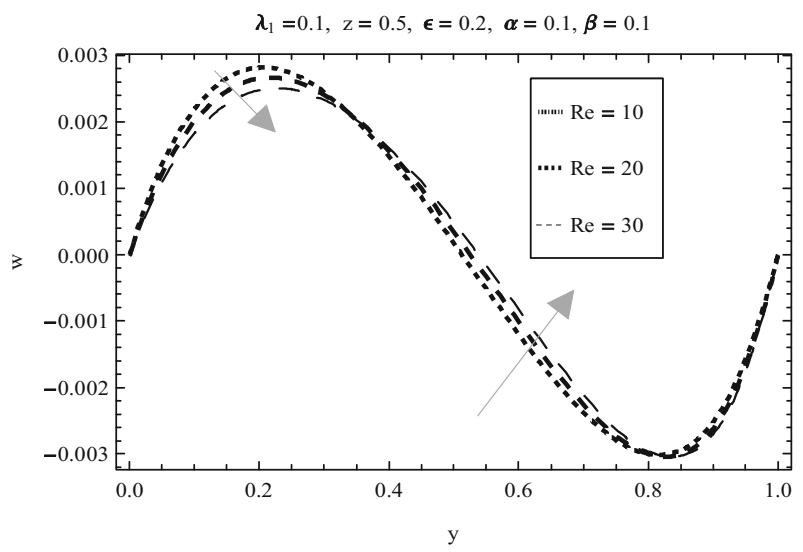

Fig. 13 Velocity component $w$ along $y$ for different values of Re

The effect of injection/suction parameter $\alpha$, the Deborah number $\beta$, the ratio of relaxation time to the retardation time $\lambda_{1}$ and Reynolds number Re on pressure are shown in Figs. 14, 15, 16 and 17, respectively. It is noted from Fig. 14 that for an increase in injection/suction parameter $\alpha$ adverse pressure increases near the stationary plate; of course, favourable pressure increases near the moving plate. It means motion of the plate with suction at the plate provide a mechanism to enhance the favourable pressure. Fig. 15 indicates that pressure decreases with increasing the Deborah number which was expected naturally. It can be shown in 


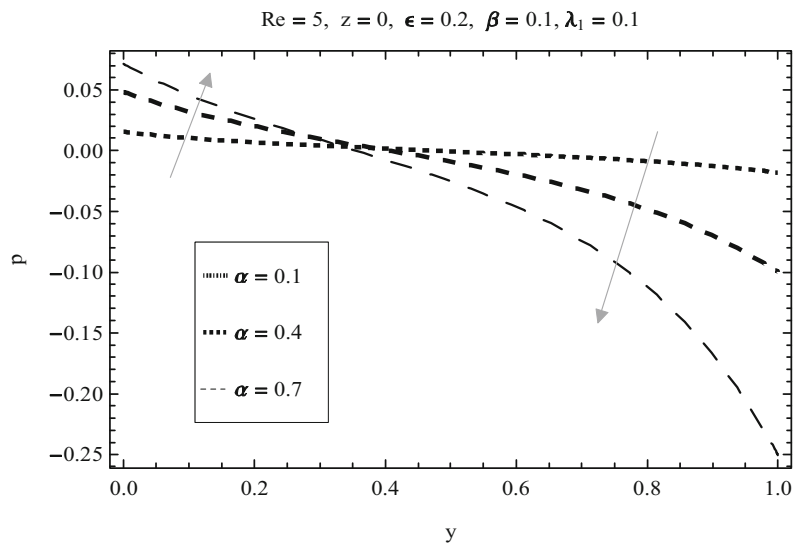

Fig. 14 Variation of $p$ along $y$ for different values of $\alpha$

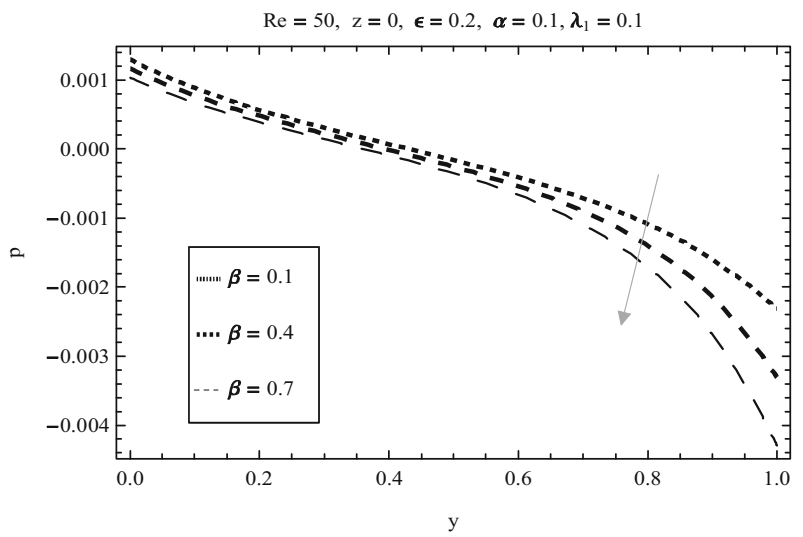

Fig. 15 Variation of $p$ along $y$ for different values of $\beta$

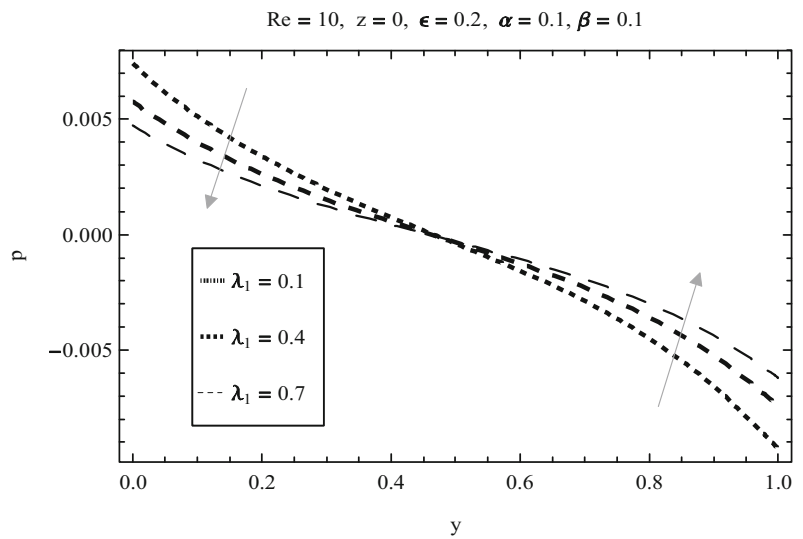

Fig. 16 Variation of $p$ along $y$ for different values of $\lambda_{1}$

Figs. 16 and 17 that there is a drop in adverse pressure from $y=0$ to about $y=0.5$, and then, onwards, there is enhancement in favourable pressure.

The variation of skin friction components at the lower plate versus Reynolds number Re in the main flow direction and transverse directions are presented in Figs. 18, 19, 20, 21, 22 and 23. Figures 18, 19 and 20 depict the effect of injection/suction, the Deborah number and the ratio of relaxation time to the retardation time on the skin friction component $F_{1}$. Figure 18 indicates that $F_{1}$ decreases with the increasing $\alpha$. Depending upon the values of $\beta$ and $\lambda_{1}, F_{1}$ increases for small values of Re and then decreases for large values of Re. Physically it seems that for large values of Re viscous forces are dominant over the inertial forces causing decrease in 


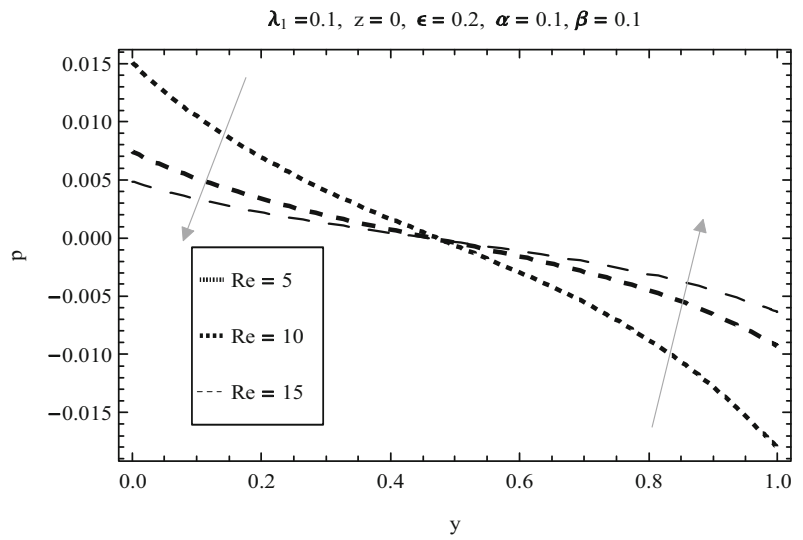

Fig. 17 Variation of $p$ along $y$ for different values of $R e$

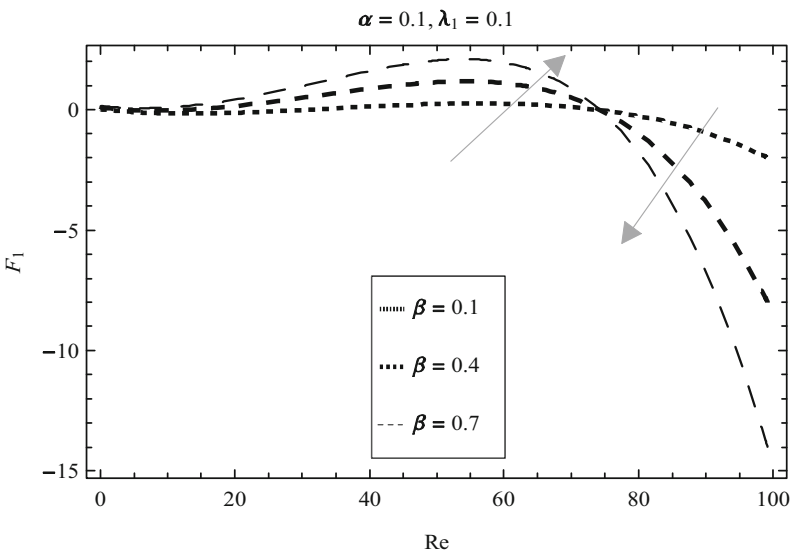

Fig. 18 Variation of $F_{1}$ along $R e$ for different values of $\beta$

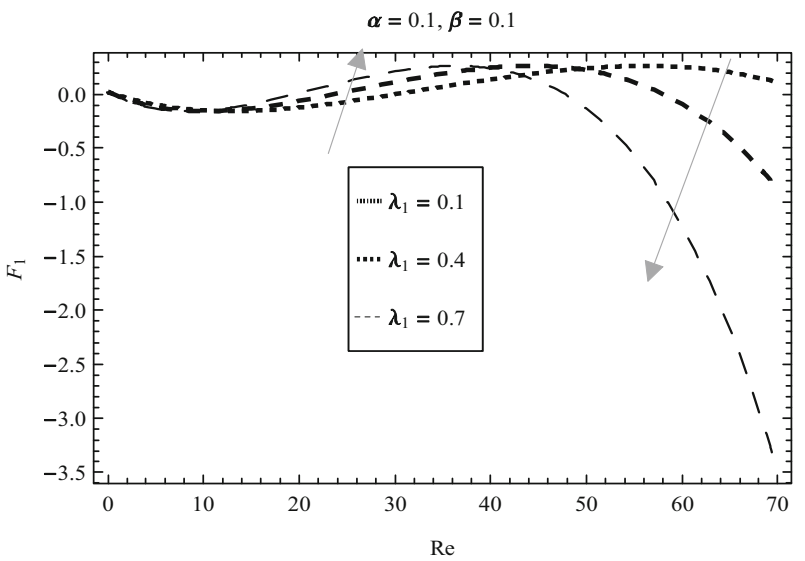

Fig. 19 Variation of $F_{1}$ along $R e$ for different values of $\lambda_{1}$

skin friction along the main flow direction and skin friction is exerted by the plate on the fluid. On the contrary, for small values of Reynolds number the inertial forces become dominant over the viscous forces resulting the change in the direction of the skin friction, that is, the skin friction is exerted by the fluid on the plate which enhances by increasing the Reynolds number. Figs. 21, 22 and 23 are drawn for skin friction component along $z$-direction versus the Reynolds number for different values of injection/suction parameter, the Deborah number and the ratio of relaxation time to the retardation time, respectively. The magnitude of skin friction component $F_{2}$ increases with the increase of injection/suction parameter. The increment in the skin friction 


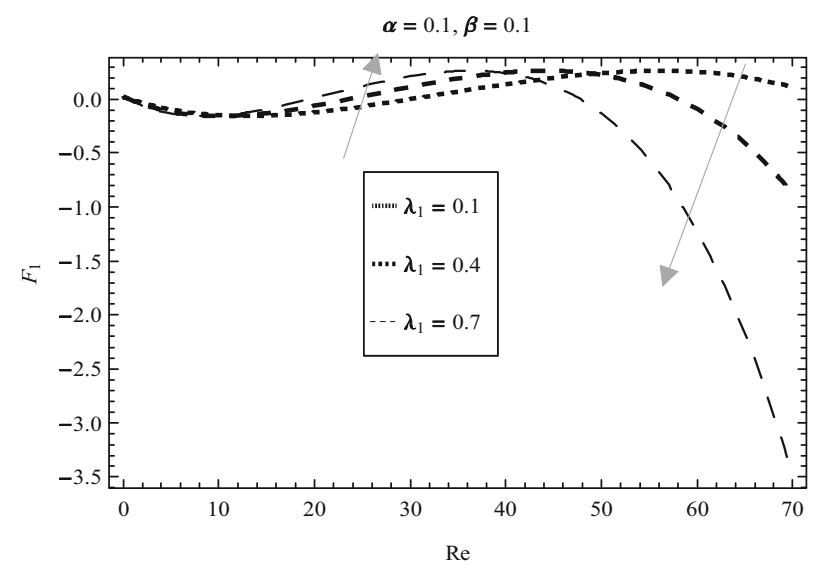

Fig. 20 Variation of $F_{1}$ along $R e$ for different values of $\lambda_{1}$

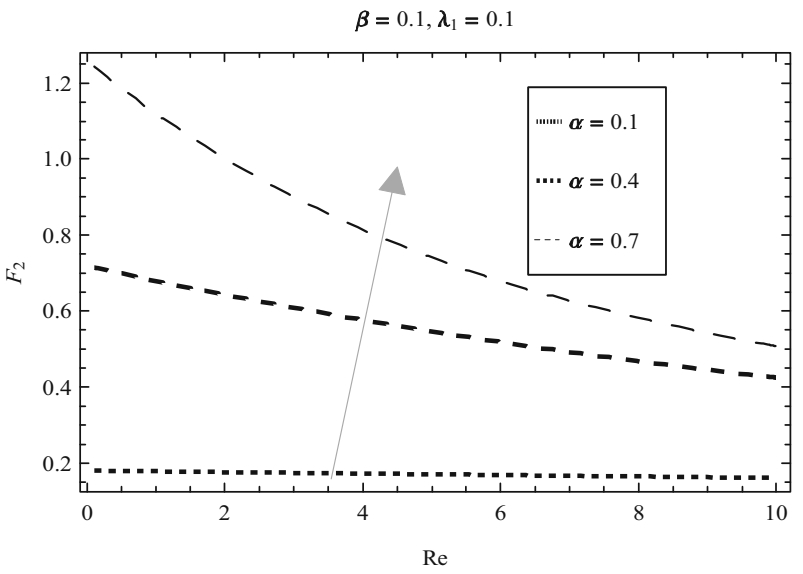

Fig. 21 Variation of $F_{2}$ along $R e$ for different values of $\alpha$

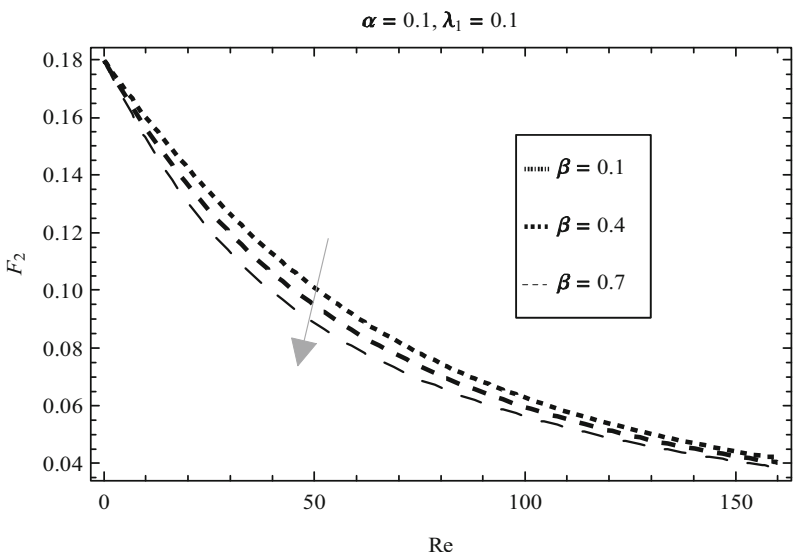

Fig. 22 Variation of $F_{2}$ along $R e$ for different values of $\beta$

exerted by the fluid on the plate for different values of Reynolds number happens due to the dominance of inertial forces over the viscous forces (Fig. 21). Moreover, it decreases with the increase in the $\beta$ and $\lambda_{1}$. The reduction in the skin friction exerted by the fluid on the plate for large values of Reynolds number happens due to the dominance of viscous forces over the inertial forces (Figs. 22, 23). 


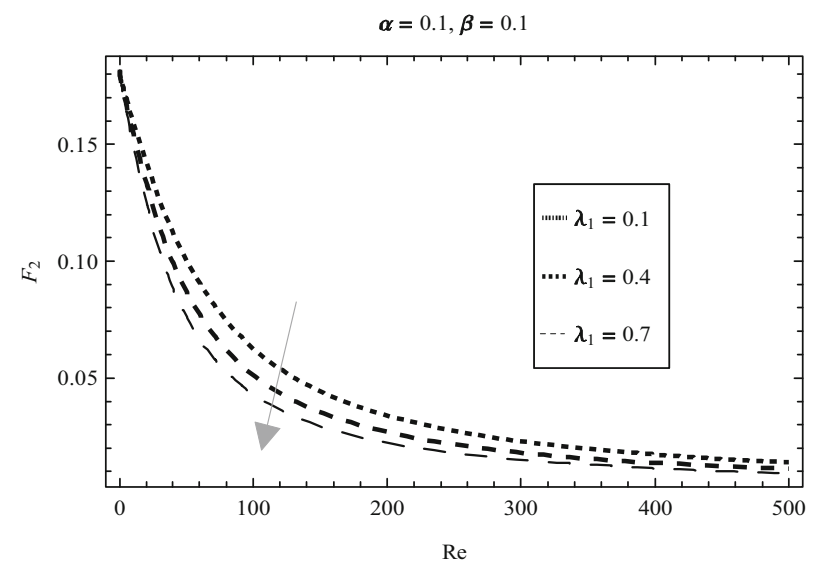

Fig. 23 Variation of $F_{2}$ along $R e$ for different values of $\lambda_{1}$

Table 1 The values of skin friction component $\left(\tau_{z}\right)$ for $\varepsilon=0.2$ and $z=0.5$

\begin{tabular}{lll}
\hline Values of $\operatorname{Re}$ & Value of $\tau_{z}$ in the paper under consideration & Values of $\tau_{z}$ in the paper [12] \\
\hline 0.1 & 0.35621585675326073 & 0.35621 \\
0.2 & 0.3528602106014592 & 0.35286 \\
0.3 & 0.3495151161671338 & 0.34951 \\
0.4 & 0.3461814949165511 & 0.34618 \\
0.5 & 0.3428602555461267 & 0.34286 \\
0.6 & 0.33955229259306274 & 0.33955 \\
0.7 & 0.33625848508717837 & 0.33625 \\
0.8 & 0.33297969525124643 & 0.33297 \\
0.9 & 0.32971676725415916 & 0.32971 \\
1.0 & 0.32647052602107657 & 0.32647 \\
\hline
\end{tabular}

\section{Conclusions}

In the light of the above discussion, the following conclusions can be drawn:

1. The main flow velocity decreases with increasing either injection/suction parameter, the ratio of relaxation time to the retardation time or Reynolds number.

2. The Deborah number enhances the main flow velocity.

3. The velocity component $v$ increases with increasing injection/suction parameter, the Deborah number, the ratio of relaxation time to the retardation time and Reynolds number.

4. The transverse component $w$ increases in forward flow and decreases in backflow with increase in injection/suction parameter. But reverse effect will be observed in case of the Deborah number, the ratio of relaxation time to the retardation time and Reynolds number.

5. Reynolds number provides a mechanism to control the skin friction components.

6. The present study offers a better result as variable injection/suction velocity is considered at both plates because in natural practice injection/suction cannot be the same in all cases.

7. The Newtonian results will be recovered when $\beta \rightarrow 0$.

\section{Appendix A}

Constants involved in this paper are

$$
\begin{aligned}
S_{1}= & \frac{\alpha R-\sqrt{(\alpha R)^{2}+4 \pi^{2}}}{2}, S_{2}=\frac{\alpha R+\sqrt{(\alpha R)^{2}+4 \pi^{2}}}{2}, \\
S_{3}= & \left(\mathrm { e } ^ { \pi } \left(-\mathrm{e}^{S_{1}} S_{1} S_{2}+\mathrm{e}^{S_{2}} S_{1} S_{2}+\mathrm{e}^{S_{1}+\pi} S_{1} S_{2}-\mathrm{e}^{S_{2}+\pi} S_{1} S_{2}+\mathrm{e}^{S_{1}} S_{1} \pi-\mathrm{e}^{S_{1}+S_{2}} S_{1} \pi-\mathrm{e}^{\pi} S_{1} \pi\right.\right. \\
& \left.+\mathrm{e}^{S_{2}+\pi} S_{1} \pi-\mathrm{e}^{S_{2}} S_{2} \pi+\mathrm{e}^{S_{1}+S_{2}} S_{2} \pi+\mathrm{e}^{\pi} S_{2} \pi-\mathrm{e}^{S_{1}+\pi} S_{2} \pi\right) \alpha /\left(-\mathrm{e}^{S_{1}} S_{1} S_{2}+\mathrm{e}^{S_{2}} S_{1} S_{2}\right.
\end{aligned}
$$




$$
\begin{aligned}
& +\mathrm{e}^{S_{1}+2 \pi} S_{1} S_{2}-\mathrm{e}^{S_{2}+2 \pi} S_{1} S_{2}+\mathrm{e}^{S_{1}} S_{1} \pi+\mathrm{e}^{S_{2}} S_{1} \pi-2 \mathrm{e}^{\pi} S_{1} \pi-2 \mathrm{e}^{S_{1}+S_{2}+\pi} S_{1} \pi+\mathrm{e}^{S_{1}+2 \pi} S_{1} \pi \\
& +\mathrm{e}^{S_{2}+2 \pi} S_{1} \pi-\mathrm{e}^{S_{1}} S_{2} \pi-\mathrm{e}^{S_{2}} S_{2} \pi+2 \mathrm{e}^{\pi} S_{2} \pi+2 \mathrm{e}^{S_{1}+S_{2}+\pi} S_{2} \pi-\mathrm{e}^{S_{1}+2 \pi} S_{2} \pi \\
& \left.-\mathrm{e}^{S_{2}+2 \pi} S_{2} \pi+\mathrm{e}^{S_{1}} \pi^{2}-\mathrm{e}^{S_{2}} \pi^{2}-\mathrm{e}^{S_{1}+2 \pi} \pi^{2}+\mathrm{e}^{S_{2}+2 \pi} \pi^{2}\right), \\
& S_{4}=-\left(\left(\begin{array}{c}
\mathrm{e}^{S_{1}} S_{1} S_{2}-\mathrm{e}^{S_{2}} S_{1} S_{2}-\mathrm{e}^{S_{1}+\pi} S_{1} S_{2}+\mathrm{e}^{S_{2}+\pi} S_{1} S_{2}+S_{1} \pi-\mathrm{e}^{S_{2}} S_{1} \pi-\mathrm{e}^{S_{1}+\pi} S_{1} \pi \\
+\mathrm{e}^{S_{1}+S_{2}+\pi} S_{1} \pi-S_{2} \pi+\mathrm{e}^{S_{1}} S_{2} \pi+\mathrm{e}^{S_{2}+\pi} S_{2} \pi-\mathrm{e}^{S_{1}+S_{2}+\pi} S_{2} \pi
\end{array}\right) \alpha\right) / \\
& \left(\begin{array}{c}
-\mathrm{e}^{S_{1}} S_{1} S_{2}+\mathrm{e}^{S_{2}} S_{1} S_{2}+\mathrm{e}^{S_{1}+2 \pi} S_{1} S_{2}-\mathrm{e}^{S_{2}+2 \pi} S_{1} S_{2}+\mathrm{e}^{S_{1}} S_{1} \pi+\mathrm{e}^{S_{2}} S_{1} \pi-2 \mathrm{e}^{\pi} S_{1} \pi \\
-2 \mathrm{e}^{S_{1}+S_{2}+\pi} S_{1} \pi+\mathrm{e}^{S_{1}+2 \pi} S_{1} \pi+\mathrm{e}^{S_{2}+2 \pi} S_{1} \pi-\mathrm{e}^{S_{1}} S_{2} \pi-\mathrm{e}^{S_{2}} S_{2} \pi+2 \mathrm{e}^{\pi} S_{2} \pi \\
+2 \mathrm{e}^{S_{1}+S_{2}+\pi} S_{2} \pi-\mathrm{e}^{S_{1}+2 \pi} S_{2} \pi-\mathrm{e}^{S_{2}+2 \pi} S_{2} \pi+\mathrm{e}^{S_{1}} \pi^{2}-\mathrm{e}^{S_{2}} \pi^{2}-\mathrm{e}^{S_{1}+2 \pi} \pi^{2}+\mathrm{e}^{S_{2}+2 \pi} \pi^{2}
\end{array}\right), \\
& S_{5}=-\left(\left(\begin{array}{c}
-S_{2} \pi-\mathrm{e}^{S_{2}} S_{2} \pi+2 \mathrm{e}^{\pi} S_{2} \pi-\mathrm{e}^{2 \pi} S_{2} \pi+2 \mathrm{e}^{S_{2}+\pi} S_{2} \pi \\
-\mathrm{e}^{S_{2}+2 \pi} S_{2} \pi+\pi^{2}-\mathrm{e}^{S_{2}} \pi^{2}-\mathrm{e}^{2 \pi} \pi^{2}+\mathrm{e}^{S_{2}+2 \pi} \pi^{2}
\end{array}\right) \alpha\right) / \\
& \left(\begin{array}{c}
\mathrm{e}^{S_{1}} S_{1} S_{2}-\mathrm{e}^{S_{2}} S_{1} S_{2}-\mathrm{e}^{S_{1}+2 \pi} S_{1} S_{2}+\mathrm{e}^{S_{2}+2 \pi} S_{1} S_{2}-\mathrm{e}^{S_{1}} S_{1} \pi-\mathrm{e}^{S_{2}} S_{1} \pi+2 \mathrm{e}^{\pi} S_{1} \pi \\
+2 \mathrm{e}^{S_{1}+S_{2}+\pi} S_{1} \pi-\mathrm{e}^{S_{1}+2 \pi} S_{1} \pi-\mathrm{e}^{S_{2}+2 \pi} S_{1} \pi+\mathrm{e}^{S_{1}} S_{2} \pi+\mathrm{e}^{S_{2}} S_{2} \pi-2 \mathrm{e}^{\pi} S_{2} \pi-2 \mathrm{e}^{S_{1}+S_{2}+\pi} S_{2} \pi \\
+\mathrm{e}^{S_{1}+2 \pi} S_{2} \pi+\mathrm{e}^{S_{2}+2 \pi} S_{2} \pi-\mathrm{e}^{S_{1}} \pi^{2}+\mathrm{e}^{S_{2}} \pi^{2}+\mathrm{e}^{S_{1}+2 \pi} \pi^{2}-\mathrm{e}^{S_{2}+2 \pi} \pi^{2}
\end{array}\right) \\
& S_{6}=-\left(\pi\left(\begin{array}{l}
-S_{1}-\mathrm{e}^{S_{1}} S_{1}+2 \mathrm{e}^{\pi} S_{1}-\mathrm{e}^{2 \pi} S_{1}+2 \mathrm{e}^{S_{1}+\pi} S_{1} \\
-\mathrm{e}^{S_{1}+2 \pi} S_{1}+\pi-\mathrm{e}^{S_{1}} \pi-\mathrm{e}^{2 \pi} \pi+\mathrm{e}^{S_{1}+2 \pi} \pi
\end{array}\right) \alpha\right) / \\
& \left(\begin{array}{c}
-\mathrm{e}^{S_{1}} S_{1} S_{2}+\mathrm{e}^{S_{2}} S_{1} S_{2}+\mathrm{e}^{S_{1}+2 \pi} S_{1} S_{2}-\mathrm{e}^{S_{2}+2 \pi} S_{1} S_{2}+\mathrm{e}^{S_{1}} S_{1} \pi+\mathrm{e}^{S_{2}} S_{1} \pi-2 \mathrm{e}^{\pi} S_{1} \pi \\
-2 \mathrm{e}^{S_{1}+S_{2}+\pi} S_{1} \pi+\mathrm{e}^{S_{1}+2 \pi} S_{1} \pi+\mathrm{e}^{S_{2}+2 \pi} S_{1}-\mathrm{e}^{S_{1}} S_{2} \pi-\mathrm{e}^{S_{2}} S_{2} \pi+2 \mathrm{e}^{\pi} S_{2} \pi \\
+2 \mathrm{e}^{S_{1}+S_{2}+\pi} S_{2} \pi-\mathrm{e}^{S_{1}+2 \pi} S_{2} \pi-\mathrm{e}^{S_{2}+2 \pi} S_{2} \pi+\mathrm{e}^{S_{1}} \pi^{2}-\mathrm{e}^{S_{2}} \pi^{2}-\mathrm{e}^{S_{1}+2 \pi} \pi^{2}+\mathrm{e}^{S_{2}+2 \pi} \pi^{2}
\end{array}\right) \\
& S_{7}=-\left(\mathrm{e}^{\pi}\left(\mathrm{e}^{S_{1}} S_{11}-\mathrm{e}^{\pi} S_{11}-\mathrm{e}^{S_{1}} S_{1} S_{11}+\mathrm{e}^{S_{1}} S_{12}-\mathrm{e}^{\pi} S_{12}-\mathrm{e}^{S_{2}} S_{1} S_{12}+\mathrm{e}^{S_{1}} S_{11} \pi+\mathrm{e}^{S_{2}} S_{12} \pi\right)\right) / \\
& \left(-S_{1}+\mathrm{e}^{2 \pi} S_{1}+\pi+\mathrm{e}^{2 \pi} \pi-2 \mathrm{e}^{S_{1}+\pi} \pi\right)-\left(\mathrm { e } ^ { \pi } \left(\mathrm{e}^{S_{2}} S_{1}-\mathrm{e}^{\pi} S_{1}-\mathrm{e}^{S_{1}} S_{2}+\mathrm{e}^{\pi} S_{2}+\mathrm{e}^{S_{1}} \pi\right.\right. \\
& \left.-\mathrm{e}^{S_{2}} \pi\right)\left(( 2 ( \mathrm { e } ^ { S _ { 1 } } - \mathrm { e } ^ { - \pi } ) \pi - ( - \mathrm { e } ^ { - \pi } + \mathrm { e } ^ { \pi } ) ( S _ { 1 } + \pi ) ) \left(2 \left(-\mathrm{e}^{S_{1}}\left(1+S_{1}\right) S_{11}-\mathrm{e}^{S_{2}}\left(1+S_{2}\right)\right.\right.\right. \\
& \left.\left.S_{12}\right) \pi-\left(-S_{11}-S_{12}\right)\left(\mathrm{e}^{-\pi} \pi+\mathrm{e}^{\pi} \pi\right)\right)-\left(-\left(-\mathrm{e}^{-\pi}+\mathrm{e}^{\pi}\right)\left(-S_{11}-S_{12}\right)-2\left(\mathrm{e}^{S_{1}} S_{11}\right.\right. \\
& \left.\left.\left.\left.+\mathrm{e}^{S_{2}} S_{12}\right) \pi\right)\left(2 \pi\left(\mathrm{e}^{S_{1}} S_{1}+\mathrm{e}^{-\pi} \pi\right)-\left(S_{1}+\pi\right)\left(\mathrm{e}^{-\pi} \pi+\mathrm{e}^{\pi} \pi\right)\right)\right)\right) /\left(\left(S_{1}-\mathrm{e}^{2 \pi} S_{1}-\pi-\mathrm{e}^{2 \pi} \pi\right.\right. \\
& \left.+2 \mathrm{e}^{S_{1}+\pi} \pi\right)\left(-\left(2\left(\mathrm{e}^{S_{2}}-\mathrm{e}^{-\pi}\right) \pi-\left(-\mathrm{e}^{-\pi}+\mathrm{e}^{\pi}\right)\left(S_{2}+\pi\right)\right)\left(2 \pi\left(\mathrm{e}^{S_{1}} S_{1}+\mathrm{e}^{-\pi} \pi\right)-\left(S_{1}+\pi\right)\right.\right. \\
& \left.\left(\mathrm{e}^{-\pi} \pi+\mathrm{e}^{\pi} \pi\right)\right)+\left(2\left(\mathrm{e}^{S_{1}}-\mathrm{e}^{-\pi}\right) \pi-\left(-\mathrm{e}^{-\pi}+\mathrm{e}^{\pi}\right)\left(S_{1}+\pi\right)\right) \\
& \left.\left.\left(2 \pi\left(\mathrm{e}^{S_{2}} S_{2}+\mathrm{e}^{-\pi} \pi\right)-\left(S_{2}+\pi\right)\left(\mathrm{e}^{-\pi} \pi+\mathrm{e}^{\pi} \pi\right)\right)\right)\right), \\
& S_{8}=-\left(S_{11}-\mathrm{e}^{S_{1}+\pi} S_{11}+\mathrm{e}^{S_{1}+\pi} S_{1} S_{11}+S_{12}-\mathrm{e}^{S_{1}+\pi} S_{12}+\mathrm{e}^{S_{2}+\pi} S_{1} S_{12}+\mathrm{e}^{S_{1}+\pi} S_{11} \pi+\mathrm{e}^{S_{2}+\pi} S_{12} \pi\right) \\
& /\left(-S_{1}+\mathrm{e}^{2 \pi} S_{1}+\pi+\mathrm{e}^{2 \pi} \pi-2 \mathrm{e}^{S_{1}+\pi} \pi\right)-\left(\left(S_{1}-\mathrm{e}^{S_{2}+\pi} S_{1}-S_{2}+\mathrm{e}^{S_{1}+\pi} S_{2}+\mathrm{e}^{S_{1}+\pi} \pi\right.\right. \\
& \left.-\mathrm{e}^{S_{2}+\pi} \pi\right)\left(( 2 ( \mathrm { e } ^ { S _ { 1 } } - \mathrm { e } ^ { - \pi } ) \pi - ( - \mathrm { e } ^ { - \pi } + \mathrm { e } ^ { \pi } ) ( S _ { 1 } + \pi ) ) \left(2\left(-\mathrm{e}^{S_{1}}\left(1+S_{1}\right) S_{11}-\mathrm{e}^{S_{2}}\left(1+S_{2}\right) S_{12}\right) \pi\right.\right. \\
& \left.-\left(-S_{11}-S_{12}\right)\left(\mathrm{e}^{-\pi} \pi+\mathrm{e}^{\pi} \pi\right)\right)-\left(-\left(-\mathrm{e}^{-\pi}+\mathrm{e}^{\pi}\right)\left(-S_{11}-S_{12}\right)+2\left(-\mathrm{e}^{S_{1}} S_{11}-\mathrm{e}^{S_{2}} S_{12}\right) \pi\right) \\
& \left.\left.\left(2 \pi\left(\mathrm{e}^{S_{1}} S_{1}+\mathrm{e}^{-\pi} \pi\right)-\left(S_{1}+\pi\right)\left(\mathrm{e}^{-\pi} \pi+\mathrm{e}^{\pi} \pi\right)\right)\right)\right) /\left(\left(S_{1}-\mathrm{e}^{2 \pi} S_{1}-\pi-\mathrm{e}^{2 \pi} \pi+2 \mathrm{e}^{S_{1}+\pi} \pi\right)\right. \\
& \left(-\left(2\left(\mathrm{e}^{S_{2}}-\mathrm{e}^{-\pi}\right) \pi-\left(-\mathrm{e}^{-\pi}+\mathrm{e}^{\pi}\right)\left(S_{2}+\pi\right)\right)\left(2 \pi\left(\mathrm{e}^{S_{1}} S_{1}+\mathrm{e}^{-\pi} \pi\right)-\left(S_{1}+\pi\right)\left(\mathrm{e}^{-\pi} \pi+\mathrm{e}^{\pi} \pi\right)\right)\right. \\
& \left.\left.+\left(2\left(\mathrm{e}^{S_{1}}-\mathrm{e}^{-\pi}\right) \pi-\left(-\mathrm{e}^{-\pi}+\mathrm{e}^{\pi}\right)\left(S_{1}+\pi\right)\right)\left(2 \pi\left(\mathrm{e}^{S_{2}} S_{2}+\mathrm{e}^{-\pi} \pi\right)-\left(S_{2}+\pi\right)\left(\mathrm{e}^{-\pi} \pi+\mathrm{e}^{\pi} \pi\right)\right)\right)\right), \\
& S_{9}=-\left(-S_{11}+\mathrm{e}^{2 \pi} S_{11}-S_{12}+\mathrm{e}^{2 \pi} S_{12}-2 \mathrm{e}^{S_{1}+\pi} S_{11} \pi-2 \mathrm{e}^{S_{2}+\pi} S_{12} \pi\right) /\left(-S_{1}+\mathrm{e}^{2 \pi} S_{1}+\pi+\mathrm{e}^{2 \pi} \pi\right. \\
& \left.-2 \mathrm{e}^{S_{1}+\pi} \pi\right)-\left(( 2 ( \mathrm { e } ^ { S _ { 2 } } - \mathrm { e } ^ { - \pi } ) \pi - ( - \mathrm { e } ^ { - \pi } + \mathrm { e } ^ { \pi } ) ( S _ { 2 } + \pi ) ) \left(\left(2\left(\mathrm{e}^{S_{1}}-\mathrm{e}^{-\pi}\right) \pi-\left(-\mathrm{e}^{-\pi}+\mathrm{e}^{\pi}\right)\right.\right.\right. \\
& \left.\left(S_{1}+\pi\right)\right)\left(2\left(-\mathrm{e}^{S_{1}}\left(1+S_{1}\right) S_{11}-\mathrm{e}^{S_{2}}\left(1+S_{2}\right) S_{12}\right) \pi-\left(-S_{11}-S_{12}\right)\left(\mathrm{e}^{-\pi} \pi+\mathrm{e}^{\pi} \pi\right)\right) \\
& -\left(-\left(-\mathrm{e}^{-\pi}+\mathrm{e}^{\pi}\right)\left(-S_{11}-S_{12}\right)+2\left(-\mathrm{e}^{S_{1}} S_{11}-\mathrm{e}^{S_{2}} S_{12}\right) \pi\right)\left(2 \pi\left(\mathrm{e}^{S_{1}} S_{1}+\mathrm{e}^{-\pi} \pi\right)-\left(S_{1}+\pi\right)\right. \\
& \left.\left.\left.\left(\mathrm{e}^{-\pi} \pi+\mathrm{e}^{\pi} \pi\right)\right)\right)\right) /\left(( 2 ( \mathrm { e } ^ { S _ { 1 } } - \mathrm { e } ^ { - \pi } ) \pi - ( - \mathrm { e } ^ { - \pi } + \mathrm { e } ^ { \pi } ) ( S _ { 1 } + \pi ) ) \left(-\left(2\left(\mathrm{e}^{S_{2}}-\mathrm{e}^{-\pi}\right) \pi\right.\right.\right. \\
& \left.-\left(-\mathrm{e}^{-\pi}+\mathrm{e}^{\pi}\right)\left(S_{2}+\pi\right)\right)\left(2 \pi\left(\mathrm{e}^{S_{1}} S_{1}+\mathrm{e}^{-\pi} \pi\right)-\left(S_{1}+\pi\right)\left(\mathrm{e}^{-\pi} \pi+\mathrm{e}^{\pi} \pi\right)\right)+\left(2\left(\mathrm{e}^{S_{1}}-\mathrm{e}^{-\pi}\right) \pi\right. \\
& \left.\left.\left.-\left(-\mathrm{e}^{-\pi}+\mathrm{e}^{\pi}\right)\left(S_{1}+\pi\right)\right)\left(2 \pi\left(\mathrm{e}^{S_{2}} S_{2}+\mathrm{e}^{-\pi} \pi\right)-\left(S_{2}+\pi\right)\left(\mathrm{e}^{-\pi} \pi+\mathrm{e}^{\pi} \pi\right)\right)\right)\right) \text {, } \\
& S_{10}=\left(( 2 ( \mathrm { e } ^ { S _ { 1 } } - \mathrm { e } ^ { - \pi } ) \pi - ( - \mathrm { e } ^ { - \pi } + \mathrm { e } ^ { \pi } ) ( S _ { 1 } + \pi ) ) \left(2\left(-\mathrm{e}^{S_{1}}\left(1+S_{1}\right) S_{11}-\mathrm{e}^{S_{2}}\left(1+S_{2}\right) S_{12}\right)\right.\right. \\
& \left.\pi-\left(-S_{11}-S_{12}\right)\left(\mathrm{e}^{-\pi} \pi+\mathrm{e}^{\pi} \pi\right)\right)-\left(-\left(-\mathrm{e}^{-\pi}+\mathrm{e}^{\pi}\right)\left(-S_{11}-S_{12}\right)+2\left(-\mathrm{e}^{S_{1}} S_{11}-\mathrm{e}^{S_{2}} S_{12}\right) \pi\right) \\
& \left.\left(2 \pi\left(\mathrm{e}^{S_{1}} S_{1}+\mathrm{e}^{-\pi} \pi\right)-\left(S_{1}+\pi\right)\left(\mathrm{e}^{-\pi} \pi+\mathrm{e}^{\pi} \pi\right)\right)\right) /\left(-\left(2\left(\mathrm{e}^{S_{2}}-\mathrm{e}^{-\pi}\right) \pi-\left(-\mathrm{e}^{-\pi}+\mathrm{e}^{\pi}\right)\left(S_{2}+\pi\right)\right)\right. \\
& \left(2 \pi\left(\mathrm{e}^{S_{1}} S_{1}+\mathrm{e}^{-\pi} \pi\right)-\left(S_{1}+\pi\right)\left(\mathrm{e}^{-\pi} \pi+\mathrm{e}^{\pi} \pi\right)\right)+\left(2\left(\mathrm{e}^{S_{1}}-\mathrm{e}^{-\pi}\right) \pi-\left(-\mathrm{e}^{-\pi}+\mathrm{e}^{\pi}\right)\left(S_{1}+\pi\right)\right)
\end{aligned}
$$




$$
\begin{aligned}
& \left.\left(2 \pi\left(\mathrm{e}^{S_{2}} S_{2}+\mathrm{e}^{-\pi} \pi\right)-\left(S_{2}+\pi\right)\left(\mathrm{e}^{-\pi} \pi+\mathrm{e}^{\pi} \pi\right)\right)\right), \\
& S_{11}=\frac{-\alpha S_{5} S_{1}\left(S_{1}^{2}-\pi^{2}\right)}{\left(S_{1}-S_{2}\right)}, S_{12}=\frac{-\alpha S_{6} S_{2}\left(S_{2}^{2}-\pi^{2}\right)}{\left(S_{2}-S_{1}\right)}, \\
& S_{13}=\frac{S_{15} \mathrm{e}^{\alpha R}}{\mathrm{e}^{\alpha R}-1}, S_{14}=\frac{-S_{15} \mathrm{e}^{\alpha R}}{\mathrm{e}^{\alpha R}-1}, S_{15}=\frac{-\alpha^{2} R}{\mathrm{e}^{\alpha R}-1}, \\
& S_{16}=\frac{1}{\mathrm{e}^{S_{1}}-\mathrm{e}^{S_{2}}}\left(\begin{array}{c}
S_{18}\left(\mathrm{e}^{S_{2}}-\mathrm{e}^{\alpha R-\pi}\right)+S_{19}\left(\mathrm{e}^{S_{2}}-\mathrm{e}^{\alpha R+\pi}\right)+ \\
S_{20}\left(\mathrm{e}^{S_{2}}-\mathrm{e}^{\alpha R+S_{1}}\right)+S_{21}\left(\mathrm{e}^{S_{2}}-\mathrm{e}^{\alpha R+S_{2}}\right)
\end{array}\right), \\
& S_{17}=\frac{1}{\mathrm{e}^{S_{2}}-\mathrm{e}^{S_{1}}}\left(\begin{array}{c}
S_{18}\left(\mathrm{e}^{S_{1}}-\mathrm{e}^{\alpha R-\pi}\right)+S_{19}\left(\mathrm{e}^{S_{1}}-\mathrm{e}^{\alpha R+\pi}\right)+ \\
S_{20}\left(\mathrm{e}^{S_{1}}-\mathrm{e}^{\alpha R+S_{1}}\right)+S_{21}\left(\mathrm{e}^{S_{1}}-\mathrm{e}^{\alpha R+S_{2}}\right)
\end{array}\right), \\
& S_{18}=\frac{\alpha R^{2} S_{3}}{\left(\mathrm{e}^{\alpha R}-1\right) S_{22}}, S_{19}=\frac{\alpha R^{2} S_{4}}{\left(\mathrm{e}^{\alpha R}-1\right) S_{23}}, S_{20}=\frac{\alpha R^{2} S_{5}}{\left(\mathrm{e}^{\alpha R}-1\right) S_{24}}, S_{21}=\frac{\alpha R^{2} S_{6}}{\left(\mathrm{e}^{\alpha R}-1\right) S_{25}}, \\
& S_{22}=\left(\alpha R-\pi-S_{1}\right)\left(\alpha R-\pi-S_{2}\right), S_{23}=\left(\alpha R+\pi-S_{1}\right)\left(\alpha R+\pi-S_{2}\right) \text {, } \\
& S_{24}=\alpha R\left(\alpha R+S_{1}-S_{2}\right), S_{25}=\left(\alpha R+S_{2}-S_{1}\right) \alpha R \text {, }
\end{aligned}
$$

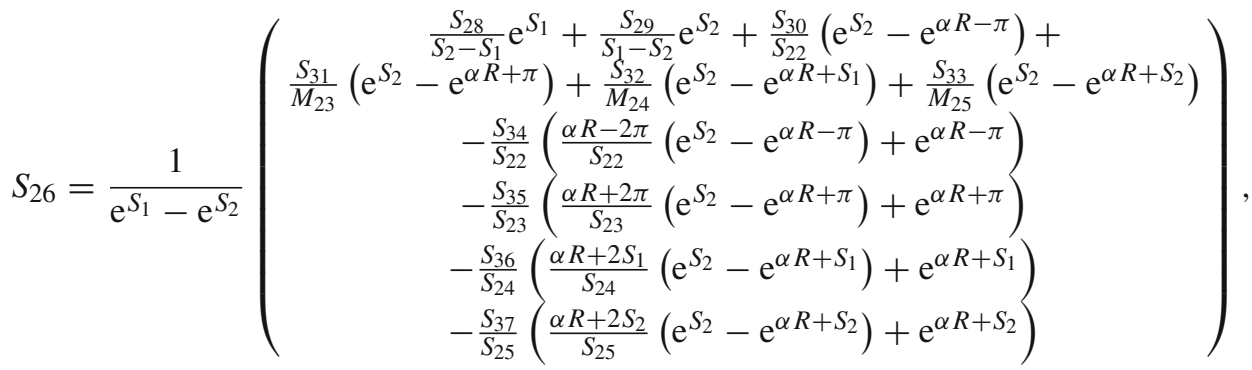

$$
\begin{aligned}
& S_{27}=\frac{1}{\mathrm{e}^{S_{2}}-\mathrm{e}^{S_{1}}}\left(\begin{array}{c}
\frac{S_{28}}{S_{2}-S_{1}} \mathrm{e}_{1}+\frac{S_{29}}{S_{1}-S_{2}} \mathrm{e}^{S_{2}}+\frac{S_{30}}{S_{22}}\left(\mathrm{e}^{S_{1}}-\mathrm{e}^{\alpha R-\pi}\right)+ \\
\frac{S_{31}}{M_{23}}\left(\mathrm{e}^{S_{1}}-\mathrm{e}^{\alpha R+\pi}\right)+\frac{S_{32}}{M_{24}}\left(\mathrm{e}^{S_{1}}-\mathrm{e}^{\alpha R+S_{1}}\right)+\frac{S_{33}}{M_{25}}\left(\mathrm{e}^{S_{1}}-\mathrm{e}^{\alpha R+S_{2}}\right) \\
-\frac{S_{34}}{S_{22}}\left(\frac{\alpha R-2 \pi}{S_{22}}\left(\mathrm{e}^{S_{1}}-\mathrm{e}^{\alpha R-\pi}\right)+\mathrm{e}^{\alpha R-\pi}\right) \\
-\frac{S_{35}}{S_{23}}\left(\frac{\alpha R+2 \pi}{S_{23}}\left(\mathrm{e}^{S_{1}}-\mathrm{e}^{\alpha R+\pi}\right)+\mathrm{e}^{\alpha R+\pi}\right) \\
-\frac{S_{36}}{S_{24}}\left(\frac{\alpha R+2 S_{1}}{S_{24}}\left(\mathrm{e}^{S_{1}}-\mathrm{e}^{\alpha R+S_{1}}\right)+\mathrm{e}^{\alpha R+S_{1}}\right) \\
-\frac{S_{37}}{S_{25}}\left(\frac{\alpha R+2 S_{2}}{S_{25}}\left(\mathrm{e}^{S_{1}}-\mathrm{e}^{\alpha R+S_{2}}\right)+\mathrm{e}^{\alpha R+S_{2}}\right)
\end{array}\right), \\
& S_{28}=-\alpha S_{1} S_{16}\left(S_{1}^{2}-\pi^{2}\right), S_{29}=-\alpha S_{2} S_{17}\left(S_{2}^{2}-\pi^{2}\right) \text {, }
\end{aligned}
$$$$
S_{30}=R\left(S_{3}\left(\alpha R S_{14}+S_{15}\right)+\frac{\alpha R S_{7}}{\mathrm{e}^{\alpha R}-1}\right)-\alpha(\alpha R-\pi)\left(S_{18}\left((\alpha R-\pi)^{2}-\pi^{2}\right)+\frac{(\alpha R)^{2} S_{3}}{\mathrm{e}^{\alpha R}-1}\right),
$$$$
S_{31}=R\left(S_{4}\left(\alpha R S_{14}+S_{15}\right)+\frac{\alpha R S_{8}}{\mathrm{e}^{\alpha R}-1}\right)-\alpha(\alpha R+\pi)\left(S_{19}\left((\alpha R+\pi)^{2}-\pi^{2}\right)+\frac{(\alpha R)^{2} S_{4}}{\mathrm{e}^{\alpha R}-1}\right),
$$$$
S_{32}=R\left(S_{5}\left(\alpha R S_{14}+S_{15}\right)+\frac{\alpha R S_{9}}{\mathrm{e}^{\alpha R}-1}\right)-\alpha\left(\alpha R+S_{1}\right)\left(S_{20}\left(\left(\alpha R+S_{1}\right)^{2}-\pi^{2}\right)+\frac{(\alpha R)^{2} S_{5}}{\mathrm{e}^{\alpha R}-1}\right),
$$$$
S_{33}=R\left(S_{6}\left(\alpha R S_{14}+S_{15}\right)+\frac{\alpha R S_{10}}{\mathrm{e}^{\alpha R}-1}\right)-\alpha\left(\alpha R+S_{2}\right)\left(S_{21}\left(\left(\alpha R+S_{2}\right)^{2}-\pi^{2}\right)+\frac{(\alpha R)^{2} S_{6}}{\mathrm{e}^{\alpha R}-1}\right),
$$$$
S_{34}=\alpha R^{2} S_{3} S_{15}, S_{35}=\alpha R^{2} S_{4} S_{15}, S_{36}=\alpha R^{2}\left(S_{5} S_{15}+\frac{S_{11}}{\mathrm{e}^{\alpha R}-1}\right),
$$$$
S_{37}=\alpha R^{2}\left(S_{6} S_{15}+\frac{S_{12}}{\mathrm{e}^{\alpha R}-1}\right), S_{38}=-\alpha S_{3}, S_{39}=-\alpha S_{4}, S_{40}=\frac{S_{5} S_{1}}{\pi^{2} R}\left(S_{1}^{2}-\alpha R S_{1}-\pi^{2}\right) \text {, }
$$$$
S_{41}=\frac{S_{6} S_{2}}{\pi^{2} R}\left(S_{2}^{2}-\alpha R S_{2}-\pi^{2}\right), S_{42}=-\alpha S_{7}, S_{43}=-\alpha S_{8},
$$ 


$$
\begin{aligned}
& S_{44}=\frac{1}{\pi^{2} R}\left(S_{9} S_{1}\left(S_{1}^{2}-\alpha R S_{1}-\pi^{2}\right)+\alpha S_{5} S_{1}^{2}\left(S_{1}^{2}-\pi^{2}\right)\right), S_{46}=\frac{S_{11} S_{1}}{\pi^{2} R}\left(S_{1}^{2}-\alpha R S_{1}-\pi^{2}\right), \\
& S_{45}=\frac{1}{\pi^{2} R}\left(S_{10} S_{2}\left(S_{2}^{2}-\alpha R S_{2}-\pi^{2}\right)+\alpha S_{6} S_{2}^{2}\left(S_{2}^{2}-\pi^{2}\right)\right), S_{47}=\frac{S_{12} S_{2}}{\pi^{2} R}\left(S_{2}^{2}-\alpha R S_{2}-\pi^{2}\right) .
\end{aligned}
$$

Open Access This article is distributed under the terms of the Creative Commons Attribution 4.0 International License (http:// creativecommons.org/licenses/by/4.0/), which permits unrestricted use, distribution, and reproduction in any medium, provided you give appropriate credit to the original author(s) and the source, provide a link to the Creative Commons license, and indicate if changes were made.

\section{References}

1. Lachmann, G.N.V.: Boundary Layer and Flow Control. Its Principles and Application, Vol. I and II. Pergamon Press, Oxford (1961)

2. Gersten, K.; Gross, J.F.: Flow and heat transfer along a plane wall with periodic suction. ZAMP 25, 399-408 (1974)

3. Singh, K.D.: Three-dimensional Couette flow with transpiration cooling. Z. Angew. Math. Phys. 50(4), 661-668 (1999)

4. Chaudhary, R.C.; Goyal, M.C.; Gupta, U.: Three-dimensional Couette flow with transpiration cooling between two horizontal parallel porous plates. J. Mech. Cont. Math. Sci. 2(1), 46-58 (2007)

5. Guria, M.; Jana, R.N.: Three-dimensional fluctuating Couette flow through the porous plates with heat transfer. Int. J. Math. Math. Sci. 61023, 01-18 (2006)

6. Sharma, B.K.; Agarwal, M.; Chaudhary, R.C.: Radiation effect on temperature distribution in three-dimensional Couette flow with suction or injection. Appl. Math. Mech. (English Edition) 28(3), 309-316 (2007)

7. Chuahan, D.S.; Kumar, V.: Heat transfer effects in the Couette flow through a composite channel partially filled with porous medium with a transverse sinusoidal injection velocity and heat source. Thermal Sci. 15(Suppl 2), S175-S186 (2011)

8. Schlichting, H.: Boundary Layer Theory. McGraw-Hill, New York (1968)

9. Singh, P.; Sharma, R.N.; Misra, J.K.; Narayan, K.A.: Three-dimensional convective flow and heat transfer in a porous medium. Indian J. Pure Appl. Math. 19(11), 1130-1135 (1988)

10. Ahmad, N.; Sarma, D.: Three-dimensional free convective flow and heat transfer through a porous medium. Indian J. Pure Appl. Math. 28(10), 1345-1353 (1997)

11. Kuznetsov, A.V.: Analytical investigation of Couette flow in a composite channel partially filled with a porous medium and partially with a clear fluid. Int. J. Heat Mass Transf. 41(16), 2556-2560 (1998)

12. Sharma, P.K.; Chaudhary, R.C.: Magnetohydrodynamics effect on three-dimensional viscous incompressible flow between two horizontal parallel porous plates and heat transfer with periodic injection/suction. IJMMS 62, 3357-3368 (2004)

13. Guria, M.; Jana, R.N.: Hydrodynamics effect on the three-dimensional flow past a vertical porous plate. Int. J. Math. Sci. 20, 3359-3372 (2005)

14. Sharma, P.K.; Saini, B.S.: The effect of injection/suction on flow past parallel plates with transpiration cooling. Turk. J. Eng. Environ. Sci. 32, 289-294 (2008)

15. Chuahan, D.S.; Kumar, V.: Three-dimensional Couette flow in a composite channel partially filled with a porous medium. Appl. Math. Sci. 4(54), 2683-2695 (2010)

16. Gireesha, B.J.; Chamkha, A.J.; Vishalakshi, C.S.; Bagewadi, C.S.: Three-dimensional Couette flow of a dusty fluid with heat transfer. Appl. Math. Model. 36, 683-701 (2012)

17. Shoaib, M.; Rana, M.A.; Siddiqui, A.M.: Three-dimensional flow of upper convected Maxwell fluid along an infinite plane wall with periodic suction. J. Comput. Theor. Nanosci. 13(8), 5247-5253 (2016)

Publisher's Note Springer Nature remains neutral with regard to jurisdictional claims in published maps and institutional affiliations. 